\title{
On the modeling of the interaction between tumor growth and the immune system using some new fractional and fractional-fractal operators
}

\author{
Behzad Ghanbari ${ }^{1,2^{*}}$ (D)
}

${ }^{*}$ Correspondence:

b.ghanbary@yahoo.com

${ }^{1}$ Department of Engineering

Science, Kermanshah University of

Technology, Kermanshah, Iran

${ }^{2}$ Department of Mathematics, Faculty of Engineering and Natural

Sciences, Bahçeşehir University,

34349 Istanbul, Turkey

\section{Springer}

\begin{abstract}
Humans are always exposed to the threat of infectious diseases. It has been proven that there is a direct link between the strength or weakness of the immune system and the spread of infectious diseases such as tuberculosis, hepatitis, AIDS, and Covid-19 as soon as the immune system has no the power to fight infections and infectious diseases. Moreover, it has been proven that mathematical modeling is a great tool to accurately describe complex biological phenomena. In the recent literature, we can easily find that these effective tools provide important contributions to our understanding and analysis of such problems such as tumor growth. This is indeed one of the main reasons for the need to study computational models of how the immune system interacts with other factors involved. To this end, in this paper, we present some new approximate solutions to a computational formulation that models the interaction between tumor growth and the immune system with several fractional and fractal operators. The operators used in this model are the Liouville-Caputo, Caputo-Fabrizio, and Atangana-Baleanu-Caputo in both fractional and fractal-fractional senses. The existence and uniqueness of the solution in each of these cases is also verified. To complete our analysis, we include numerous numerical simulations to show the behavior of tumors. These diagrams help us explain mathematical results and better describe related biological concepts. In many cases the approximate results obtained have a chaotic structure, which justifies the complexity of unpredictable and uncontrollable behavior of cancerous tumors. As a result, the newly implemented operators certainly open new research windows in further computational models arising in the modeling of different diseases. It is confirmed that similar problems in the field can be also be modeled by the approaches employed in this paper.
\end{abstract}

Keywords: Mathematical modeling; Fractional and fractional-fractal derivatives; Tumor growth modeling; The immune system; Numerical simulations; Sensitivity analysis (c) The Author(s) 2020. This article is licensed under a Creative Commons Attribution 4.0 International License, which permits use, sharing, adaptation, distribution and reproduction in any medium or format, as long as you give appropriate credit to the original author(s) and the source, provide a link to the Creative Commons licence, and indicate if changes were made. The images or other third party material in this article are included in the article's Creative Commons licence, unless indicated otherwise in a credit line to the material. If material is not included in the article's Creative Commons licence and your intended use is not permitted by statutory regulation or exceeds the permitted use, you will need to obtain permission directly from the copyright holder. To view a copy of this licence, visit http://creativecommons.org/licenses/by/4.0/. 


\section{Introduction}

The immune system is a real masterpiece that does extraordinary things every day while we do not realize such numerous activities. The job of the immune system is protecting our bodies against invading factors such as bacteria and viruses. Many factors such as low nutrient intake, lack of sleep, and high stress weaken a person's immune system. On the other hand, people with cancer are also at risk for infectious diseases since they usually undergo special chemotherapy treatments using special drugs that weaken their immune system. The main purpose of these special drugs is destroying diseased cells without damaging adjacent tissues. Unfortunately, some healthy cells and tissues in the body may also be affected during this treatment.

Nowadays, with the pandemic outbreak of the dangerous infectious disease COVID-19 all over the world, infectious disease specialists and epidemiologists constantly emphasize the strengthening of the immune system as one of the most important ways to prevent this deadly disease.

In this paper, we provide some novel approximate solutions to a computational model that formulates the interaction between tumor growth and the immune system, including several fractional and fractal operators. The model consists of three state variables, each representing the number of specific cells in the problem. The interaction between these variables is presented by Itik and Banks [26] through following nonlinear differential equation system:

$$
\begin{aligned}
& \frac{d \mathrm{~T}(\tau)}{d \tau}=k_{1}^{\prime} \mathrm{T}(\tau)\left(1-\frac{\mathrm{T}(\tau)}{s_{1}}\right)-\beta_{12}^{\prime} \mathrm{T}(\tau) \mathrm{H}(\tau)-\beta_{13}^{\prime} \mathrm{T}(\tau) \mathrm{E}(\tau), \\
& \frac{d \mathrm{H}(\tau)}{d \tau}=k_{2}^{\prime} \mathrm{H}(\tau)\left(1-\frac{\mathrm{H}(\tau)}{s_{2}}\right)-\beta_{21}^{\prime} \mathrm{T}(\tau) \mathrm{H}(\tau), \\
& \frac{d \mathrm{E}(\tau)}{d \tau}=\frac{k_{3} \mathrm{~T}(\tau) \mathrm{E}(\tau)}{\mathrm{T}(\tau)+s_{3}}-\beta_{31}^{\prime} \mathrm{T}(\tau) \mathrm{E}(\tau)-c_{3}^{\prime} \mathrm{E}(\tau),
\end{aligned}
$$

subject to initial conditions $(T(0), H(0), E(0))=\left(T_{0}, H_{0}, E_{0}\right) \geq 0$.

In this model, $\mathrm{T}(\tau)$ is used to count the number of tumor cells at time $\tau, \mathrm{H}(\tau)$ represents the number of healthy host cells, and $\mathrm{E}(\tau)$ refers to the number of effector immune cells in the single tumor-site compartment. Moreover, $\tau$ represents the rate of change in the population of the tumor cells, $k_{1}^{\prime}$ describes the growth of the tumor cells, and $s_{1}^{\prime}$ is their maximum carrying capacity. The first term of Eq. (1) expresses to the logistic growth of the tumor cells in the absence of any effect from other cell populations. The competition between the host cells $\mathrm{H}(\tau)$ and the tumor cells $\mathrm{T}(\tau)$, which results in the loss of the tumor cell population, is given by the term $\beta_{12}^{\prime} \mathrm{T}(\tau) \mathrm{H}(\tau)$. Moreover, $\beta_{12}^{\prime}$ refers to the tumor cell killing rate by the effector cells $\mathrm{E}(\tau)$. In the second equation of system (1) the healthy tissue cells also grow logistically with the growth rate $k_{2}^{\prime}$ and maximum carrying capacity $s_{2}^{\prime}$. We assume that the cancer cells proliferate faster than the healthy cells, and thus $k_{1}^{\prime}>k_{2}^{\prime}$. The tumor cells also inactivate the healthy cells at the rate $\beta_{21}^{\prime}$. Also, the third equation of system (1) illustrates the stimulation of the immune system by the tumor cells with tumor specific antigens. One of the other assumptions considered in the model is that the immune system depends directly on the number of tumor cells a the rate of positive constants $k_{3}^{\prime}$ and $s_{3}^{\prime}$. Finally, $\beta_{31}^{\prime}$ is the rate of change corresponding to the inactivated effector cells by the tumor cells, and $c_{3}^{\prime}$ is their rate of natural death. All parameters in the model are positive constants. 
For simplicity of analysis, we first nondimensionalize system (1) by employing the definitions

$$
\mathcal{T}(t)=\frac{\mathrm{T}(\tau)}{s_{1}}, \quad \mathcal{H}(t)=\frac{\mathrm{H}(\tau)}{s_{2}}, \quad \mathcal{E}(t)=\frac{\mathrm{E}(\tau)}{s_{3}}, \quad t=k_{1} \tau .
$$

In addition, we use the following new parameters:

$$
\begin{aligned}
& \beta_{31}=\frac{\beta_{31}^{\prime} s_{1}}{k_{1}}, \quad \beta_{13}=\frac{\beta_{13}^{\prime} s_{3}}{k_{1}}, \quad \beta_{12}=\frac{\beta_{12}^{\prime} s_{2}}{k_{1}}, \quad \beta_{31}=\frac{\beta_{31}^{\prime} s_{1}}{k_{1}}, \\
& k_{2}=\frac{k_{2}^{\prime}}{k_{1}}, \quad k_{3}=\frac{k_{3}^{\prime}}{k_{1}}, \quad s_{3}=\frac{s_{3}^{\prime}}{s 1}, \quad c_{3}=\frac{c_{3}^{\prime}}{k_{1}} .
\end{aligned}
$$

By involving the introduced changes in equations (2) and (3), we obtain a new dimensionless form for the problem [26]:

$$
\begin{aligned}
\frac{d \mathcal{T}(t)}{d t} & =\mathcal{T}(t)(1-\mathcal{T}(t))-\beta_{12} \mathcal{T}(t) \mathcal{H}(t)-\beta_{13} \mathcal{T}(t) \mathcal{E}(t) \\
\frac{d \mathcal{H}(t)}{d t} & =k_{2} \mathcal{H}(t)(1-\mathcal{H}(t))-\beta_{21} \mathcal{T}(t) \mathcal{H}(t) \\
\frac{d \mathcal{E}(t)}{d t} & =\frac{k_{3} \mathcal{T}(t) \mathcal{E}(t)}{\mathcal{T}(t)+s_{3}}-\beta_{31} \mathcal{T}(t) \mathcal{E}(t)-c_{3} \mathcal{E}(t)
\end{aligned}
$$

Due to the great importance of model (4), many researchers have shown interest in examining various technical and computational aspects of this model. The authors explain the biological relevance of model (7). In [41] the authors have utilized the localization of compact invariant sets (LCIS) method and Lyapunov stability theory to investigate the global dynamics of model (4). In [28] the authors have focused on the mathematical points of view, such as the existence of Hopf bifurcations corresponding to the model. In [24] the authors considered the model using the Caputo-Fabrizio-Caputo and new fractional derivative with Mittag-Leffler kernel in the Liouville-Caputo sense. Starko and Coria [40] provided sufficient conditions on model parameters and treatment parameters under which all trajectories in the positive orthant tend to the tumor-free equilibrium point. The authors in [32] have developed an efficient methodology of partial control and applied it to avoid the extinction of the healthy tissue. In [42], taking impulsive differential equations into account, the authors have presented a mathematical formulation of tumor-immune interaction with periodically pulsed immunotherapy. In [3], model (4) was modified to include three delay parameters in the problem.

Fractional calculus has a relatively long history almost as long as a integer-order differential account. However, in recent decades, the implementations of these concepts was neglected compared to standard calculus. This trend seems to have changed in the past years in general. A defining sign of this change is the increasing use of these tools in the literature. Thanks to the researchers' efforts, many differentiated and integral operators based on different approaches were proposed and then successfully implemented in the past few years $[1,4,5,10,13,23,25,30]$. From the perspective of numerical aspects, a wide range of new mathematical methods was successfully applied in various branches of science $[6,13,16-19,27,29,35,37,39]$. For example, in [9] the authors have developed an efficient numerical treatment for ordinary fractional and fractal-fractional differential 
differential equations, which is based on Newton polynomials. In [8], Atangana and his collaborator have successfully applied a new numerical algorithm to approximate a modified version of the Chua attractor model with both fractional and fractal-fractional operators. An efficient numerical technique, the Atangana-Seda numerical scheme, based on Newton polynomials, is utilized in [7] to handle a chaotic problem with fractional operators, which include the exponential decay, power law, and Mittag-Leffler kernel. In [23] the author has employed some novel differential and integral operators of fractional order and fractal dimension using he uville-Caputo and Atangana-Baleanu definitions to obtain multiple attractors and periodicity on the Vallis model for El Niño/La Niña-Southern oscillation model. A new fractional-order compartmental SEIRS model with Caputo-type fractional-order derivative was also studied in [25]. Chaotic systems are almost one of the most important and applicable types of nonlinear equations [12, 21, 34, 36, 38]. Therefore in many cases the exact solution is not available for such equations. On the other hand, the use of new derivative operators in structures of chaotic systems has made significant development in this field [2, 22]. In some cases the researchers have obtained desirable attractors, which were not achievable by common integer-order operators. This fact highlights the importance of new derivative operators in other real-world models. Motivated by these achievements, especially following the work [24], we intend to investigate the model presented in equation (4) using some new efficient fractional and fractionalfractional operators.

To reach this goal, the subsequent parts of the paper are structured as follows. The analysis of model equilibrium points is presented in Sect. 2. This model is examined in the next section via the Liouville-Caputo fractional-order derivative. This section also confirms that under appropriate assumptions, the model always possesses a unique solution. Then a numerical method corresponding to this structure is designed and then utilized. Besides, detailed numerical simulations are presented. Similar processes will be followed in Sects. 4 and 5 of the paper, with the Caputo-Fabrizio-Caputo and Atangana-BaleanuCaputo fractional derivative operators, respectively. In Sect. 6, we examine the model via several fractal-fractional operators. This section also presents the numerical methods corresponding to each of these operators. To investigate the dynamic behavior of the results, we added several numerical simulations. Finally, in Sect. 7, we present a summary of the results and achievements of the paper.

\section{Investigation of stability of equilibrium points of models}

In this section, we analyze the equilibrium points of the considered model (4). These points are in fact the roots of the nonlinear algebraic system that is contracted on the right-hand side of the model. By solving this system, we determine six possible equilibrium points for the model [28].

Point 1: the no "living cell" singular point $\mathcal{P}_{1}=(0,0,0)$.

Point 2: The tumor-free fixed point $\mathcal{P}_{2}=(0,1,0)$.

Point 3: The fixed point $\mathcal{P}_{3}=(1,0,0)$, which implies the existence of tumor cells in the model.

Point 4: The fixed point $\mathcal{P}_{4}=\left(\mathcal{T}^{*}, 0, \frac{1-\mathcal{T}^{*}}{\beta_{13}}\right)$. The first coordinate $\mathcal{T}^{*}$ is determined by finding the nonnegative root(s) of the characteristic equation

$$
\beta_{13} \mathcal{T}^{* 2}+\mathcal{T}^{*}\left(c_{3}+s_{3} \beta_{31}-k_{3}\right)+s_{3} c_{3}=0
$$


This equation has the acceptable root

$$
\mathcal{T}^{*}=\frac{k_{3}-c_{3}-s_{3} \beta_{31}+\sqrt{\left(k_{3}-c_{3}-s_{3} \beta_{31}\right)^{2}-4 s_{3} c_{3} \beta_{31}}}{2 \beta_{31}} .
$$

Necessary conditions for the existence of this equilibrium point are

$$
k_{3}>c_{3}+s_{3} \beta_{31}, \quad \mathcal{T}^{*}<1
$$

Point 5: The singular point $\mathcal{P}_{5}=\left(\frac{k 2\left(\beta_{12}-1\right)}{\beta 12 \beta 21-k 2}, \frac{\beta 21-k 2}{\beta_{12} \beta_{21}-k 2}, 0\right)$, which implies the coexistence of cancer and host cells. The necessary conditions for the existence of this equilibrium point are

$$
\beta_{21}>k_{2}, \quad \beta_{12}>1, \quad \beta_{12} \beta_{21}>k_{2} .
$$

It should also be noted that for $\beta_{12}=1$, this equilibrium point becomes the equilibrium point $\mathcal{P}_{2}$.

Point 6: The interior fixed point $\mathcal{P}_{6}=\left(\mathcal{T}^{*}, \frac{k_{2}-\beta_{21} \mathcal{T}^{*}}{k_{2}}, \frac{k_{2}\left(1-\beta_{12}\right)+\mathcal{T}^{*}\left(\beta_{21} \beta_{12}-k_{2}\right)}{k_{2} \beta_{13}}\right)$, where $\mathcal{T}^{*}$ is a positive root of $\beta_{13} \mathcal{T}^{* 2}+\mathcal{T}^{*}\left(c_{3}+s_{3} \beta_{31}-k_{3}\right)+s_{3} c_{3}=0$, as mentioned earlier. In this case, all three cell populations are present in the problem. The necessary conditions for the existence of this equilibrium point are

$$
k_{3}>c_{3}+s_{3} \beta_{31}, \quad \beta_{12}<1, \quad \beta_{12} \beta_{21}>k_{2}, \quad \beta_{21}>k_{2}, \quad \mathcal{T}^{*}<\frac{k_{2}}{\beta_{21}} .
$$

Moreover, the Jacobi matrix corresponding to this system is

$$
\begin{aligned}
J\left(\mathcal{T}^{*}, \mathcal{H}^{*}, \mathcal{E}^{*}\right) & \\
\quad= & {\left[\begin{array}{ccc}
-\beta_{12} \mathcal{H}^{*}-\beta_{13} \mathcal{E}^{*}-2 \mathcal{T}^{*}+1 & -\beta_{12} \mathcal{T}^{*} & -\beta_{13} \mathcal{T}^{*} \\
-\mathcal{H}^{*} \beta_{21} & k_{2}\left(1-\mathcal{H}^{*}\right)-k_{2} \mathcal{H}^{*}-\beta 21 \mathcal{T}^{*} & 0 \\
\frac{k_{3} \mathcal{E}^{*}}{\mathcal{T}^{*}+s_{3}}-\frac{k_{3} \mathcal{T}^{*} \mathcal{E}^{*}}{\left(\mathcal{T}^{*}+s_{3}\right)^{2}}-\beta_{31} \mathcal{E}^{*} & 0 & \frac{k_{3} \mathcal{T}^{*}}{\mathcal{T}^{*}+s_{3}}-\beta_{31} \mathcal{T}^{*}-c_{3}
\end{array}\right] . }
\end{aligned}
$$

\section{The model via the Liouville-Caputo fractional derivative}

In this section, we consider model (4) with the Liouville-Caputo (LC) fractional derivative,

$$
\begin{aligned}
& { }_{0}^{\mathrm{LC}} \mathcal{D}_{t}^{\alpha} \mathcal{T}(t)=\mathcal{T}(t)(1-\mathcal{T}(t))-\beta_{12} \mathcal{T}(t) \mathcal{H}(t)-\beta_{13} \mathcal{T}(t) \mathcal{E}(t) \\
& { }_{0}^{\mathrm{LC}} \mathcal{D}_{t}^{\alpha} \mathcal{H}(t)=k_{2} \mathcal{H}(t)(1-\mathcal{H}(t))-\beta_{21} \mathcal{T}(t) \mathcal{H}(t) \\
& { }_{0}^{\mathrm{LC}} \mathcal{D}_{t}^{\alpha} \mathcal{E}(t)=\frac{k_{3} \mathcal{T}(t) \mathcal{E}(t)}{\mathcal{T}(t)+s_{3}}-\beta_{31} \mathcal{T}(t) \mathcal{E}(t)-c_{3} \mathcal{E}(t)
\end{aligned}
$$

where the LC fractional derivative is defined as [14]

$$
{ }_{0}^{\mathrm{LC}} \mathcal{D}_{t}^{\alpha} \mathcal{T}(t)=\frac{1}{\Gamma(\alpha)} \int_{0}^{t}(t-\eta)^{\alpha-1} \phi^{\prime}(\eta) d \eta, \quad 0<\boldsymbol{\alpha} \leq 1
$$


Utilizing the Laplace transform on the LC derivative (8), we get

$$
\mathcal{L}\left\{{ }_{0}^{\mathrm{LC}} \mathcal{D}_{t}^{\alpha} \phi(t)\right\}=s^{\alpha} \mathcal{L}\{\phi(t)\}-\sum_{k=0}^{m-1} s^{\alpha-k-1} \phi^{(k)}(0), \quad m=\lceil\boldsymbol{\alpha}\rceil .
$$

Taking (9) into account and then utilizing the inverse Laplace transform on Eq. (7), we get

$$
\begin{aligned}
& \mathcal{T}(t)=\mathcal{T}(0)+\mathcal{L}^{-1}\left\{\frac{1}{s^{\alpha}} \mathcal{L}\left[\mathcal{T}(t)(1-\mathcal{T}(t))-\beta_{12} \mathcal{T}(t) \mathcal{H}(t)-\beta_{13} \mathcal{T}(t) \mathcal{E}(t)\right]\right\} \\
& \mathcal{H}(t)=\mathcal{H}(0)+\mathcal{L}^{-1}\left\{\frac{1}{s^{\alpha}} \mathcal{L}\left[k_{2} \mathcal{H}(t)(1-\mathcal{H}(t))-\beta_{21} \mathcal{T}(t) \mathcal{H}(t)\right]\right\} \\
& \mathcal{E}(t)=\mathcal{E}(0)+\mathcal{L}^{-1}\left\{\frac{1}{s^{\alpha}} \mathcal{L}\left[\frac{k_{3} \mathcal{T}(t) \mathcal{E}(t)}{\mathcal{T}(t)+s_{3}}-\beta_{31} \mathcal{T}(t) \mathcal{E}(t)-c_{3} \mathcal{E}(t)\right]\right\}
\end{aligned}
$$

From (10) we suggest the following iterative schemes:

$$
\begin{aligned}
& \mathcal{T}_{n}(t)=\mathcal{T}_{0}+\mathcal{L}^{-1}\left\{\frac{1}{s^{\alpha}} \mathcal{L}\left[\mathcal{T}_{n-1}(t)\left(1-\mathcal{T}_{n-1}(t)\right)-\beta_{12} \mathcal{T}_{n-1}(t) \mathcal{H}_{n-1}(t)-\beta_{13} x_{n-1}(t) \mathcal{E}_{n-1}(t)\right]\right\} \\
& \mathcal{H}_{n}(t)=\mathcal{H}_{0}+\mathcal{L}^{-1}\left\{\frac{1}{s^{\alpha}} \mathcal{L}\left[k_{2} \mathcal{H}_{n-1}(t)\left(1-\mathcal{H}_{n-1}(t)\right)-\beta_{21} \mathcal{T}_{n-1}(t) y_{n-1}(t)\right]\right\} \\
& \mathcal{E}_{n}(t)=\mathcal{E}_{0}+\mathcal{L}^{-1}\left\{\frac{1}{s^{\alpha}} \mathcal{L}\left[\frac{k_{3} \mathcal{T}_{n-1}(t) \mathcal{E}_{n-1}(t)}{\mathcal{T}_{n-1}(t)+s_{3}}-\beta_{31} \mathcal{T}_{n-1}(t) \mathcal{E}_{n-1}(t)-c_{3} \mathcal{E}(t)\right]\right\}
\end{aligned}
$$

where

$$
\mathcal{T}_{0}=\mathcal{T}(0), \quad \mathcal{H}_{0}=\mathcal{H}(0), \quad \mathcal{E}_{0}=\mathcal{E}(0)
$$

The desired approximate solutions can be obtained by computing the limits

$$
\mathcal{T}(t)=\lim _{n \rightarrow \infty} \mathcal{T}_{n}(t), \quad \mathcal{H}(t)=\lim _{n \rightarrow \infty} \mathcal{H}_{n}(t), \quad \mathcal{E}(t)=\lim _{n \rightarrow \infty} \mathcal{E}_{n}(t)
$$

\subsection{Existence and uniqueness}

Let us assume that a Banach space like $\Omega$ has a closed convex bounded subset $\Xi$ that contains a fixed point of $\Omega$. Moreover, let $\omega: \Xi \rightarrow \Xi$ be a condensing map. Moreover, let us assume that there exists $\Delta \in(0, \xi)$ such that $\mathfrak{X}, \mathfrak{X}, \mathfrak{Z} \in L_{1 / \Delta}\left([0, T], R^{+}\right)$. The exist functions $\mathfrak{X}^{*}, \mathfrak{X}^{*}, \mathfrak{Z}^{*} \in(R, \Omega) \cap L_{\text {loc }}^{1}(R, \Omega)$ such that $\mathfrak{X}=\mathfrak{X}^{*}+\mathfrak{X}^{* *}, \mathfrak{X}=\mathfrak{X}^{*}+\mathfrak{X}^{* *}$, and $\mathfrak{Z}=\mathfrak{Z}^{*}+\mathfrak{J}^{* *}$. Then we have:

1. $\mathfrak{X}^{*}, \mathfrak{X}^{*}$, and $\mathfrak{Z}^{*}$ are Lipschitz and bounded.

2. $\mathfrak{X}^{* *}, \mathfrak{X}^{* *}$, and $\mathfrak{క}^{* *}$ are compact and bounded.

3. $|R(t, m)-R(t, z)| \leq L_{1}(t)\|m-z\|$.

With the help of the Riemann-Liouville integral, Eq. (7) is written as

$$
\begin{aligned}
& \mathfrak{X}(t)=\mathfrak{X}(0)+\frac{1}{\Gamma(\rho)} \int_{0}^{t}(t-\eta)^{\rho-1} \mathfrak{X}^{*}(\eta, \mathfrak{X}(\eta)) d \eta+\frac{1}{\Gamma(\rho)} \int_{0}^{t}(t-\eta)^{\rho-1} \mathfrak{X}^{* *}(\eta, \mathfrak{X}(\eta)) d \eta, \\
& \mathfrak{X}(t)=\mathfrak{X}(0)+\frac{1}{\Gamma(\rho)} \int_{0}^{t}(t-\eta)^{\rho-1} \mathfrak{X}^{*}(\eta, \mathfrak{X}(\eta)) d \eta+\frac{1}{\Gamma(\rho)} \int_{0}^{t}(t-\eta)^{\rho-1} \mathfrak{X}^{* *}(\eta, \mathfrak{X}(\eta)) d \eta, \\
& \mathfrak{z}(t)=\mathfrak{Z}(0)+\frac{1}{\Gamma(\rho)} \int_{0}^{t}(t-\eta)^{\rho-1} \mathfrak{z}^{*}(\eta, \mathfrak{Z}(\eta)) d \eta+\frac{1}{\Gamma(\rho)} \int_{0}^{t}(t-\eta)^{\rho-1} \mathfrak{J}^{* *}(\eta, \mathfrak{J}(\eta)) d \eta .
\end{aligned}
$$


Theorem 1 As soon as

$$
\psi=\frac{v\|L\|_{1 / \nabla} T^{M}}{\Gamma(\rho)}<1
$$

then under two properties 1 and 2, the existence of a solution to the problem is guaranteed.

Proof Let us take $\chi$ such that $\tau(0)+\frac{1}{\Gamma(\rho)} v\left(\left\|H_{1}\right\|_{1 / \nabla}+\left\|H_{2}\right\|_{1 / \nabla}\right) T^{M} \leq \chi$, and let $\Xi_{\mu}=\{m$ : $\|m\| \leq \chi\}$ be a closed set in the Banach space $([0, T], \Omega)$ equipped with the norm of sup $\|\cdot\|$.

Then, by the definition $m: \Xi_{\chi} \rightarrow([0, T], \Omega), m \rightarrow \mathfrak{X}^{*} m+\mathfrak{X}^{* *} m$, we have

$$
\begin{aligned}
& \mathfrak{X}^{*}(t)=\mathfrak{X}^{*}(0)+\frac{1}{\Gamma(\rho)} \int_{0}^{t}(t-\eta)^{\rho-1} \mathfrak{X}^{*}(\eta, m(\eta)) d \eta, \\
& \mathfrak{X}^{* *}(t)=\mathfrak{X}^{* *}(0)+\frac{1}{\Gamma(\rho)} \int_{0}^{t}(t-\eta)^{\rho-1} \mathfrak{X}^{* *}(\eta, m(\eta)) d \eta, \\
& \mathfrak{W}^{*}(t)=\mathcal{L}^{*}(0)+\frac{1}{\Gamma(\rho)} \int_{0}^{t}(t-\eta)^{\rho-1} \mathcal{L}^{*}(\eta, m(\eta)) d \eta, \\
& \mathfrak{W}^{* *}(t)=\mathfrak{X}^{* *}(0)+\frac{1}{\Gamma(\rho)} \int_{0}^{t}(t-\eta)^{\rho-1} \mathfrak{X}^{* *}(\eta, m(\eta)) d \eta \text {, } \\
& \boldsymbol{\Xi}^{*}(t)=\boldsymbol{\Xi}^{*}(0)+\frac{1}{\Gamma(\rho)} \int_{0}^{t}(t-\eta)^{\rho-1} \boldsymbol{\Xi}^{*}(\eta, m(\eta)) d \eta, \\
& \boldsymbol{3}^{* *}(t)=\boldsymbol{3}^{* *}(0)+\frac{1}{\Gamma(\rho)} \int_{0}^{t}(t-\eta)^{\rho-1} \boldsymbol{3}^{* *}(\eta, m(\eta)) d \eta \text {. }
\end{aligned}
$$

Hence we obtain that $\mathfrak{X}, \mathfrak{X}, \mathfrak{3}$, and $\mathfrak{w}$ are condensing, and the fixed points of $\mathfrak{X}, \mathfrak{Z}$, $\mathfrak{3}$, and $\mathfrak{w}$ are verified.

I) Let us show that $\mathfrak{X}\left(\Xi_{\chi}\right) \subset \Xi_{\chi}$. For $m \in \Xi_{\chi}$, we have

$$
\begin{aligned}
\|\mathfrak{X}(t)\| \leq & \|\mathfrak{X}(0)\|+\frac{1}{\Gamma(\rho)} \int_{0}^{t}(t-\eta)^{\rho-1} \mathfrak{X}^{*}(\eta, m(\eta)) d \eta \\
\leq & \|\mathfrak{X}(0)\|+\frac{1}{\Gamma(\rho)} \int_{0}^{t}(t-\eta)^{\rho-1} \mathfrak{X}^{*}(\eta, m(\eta)) d \eta \\
& +\frac{1}{\Gamma(\rho)} \int_{0}^{t}(t-\eta)^{\rho-1} \mathfrak{X}^{* *}(\eta, m(\eta)) d \eta \\
\leq & \|\mathfrak{X}(0)\|+\frac{1}{\Gamma(\rho)}\left(\int_{0}^{t}(t-\eta)^{\frac{\rho-1}{1-\nabla}} d \eta\right)^{1-\nabla}\left(\int_{0}^{t} G_{1}^{1 / \nabla}(\eta) d \eta\right)^{\nabla} \\
& +\frac{1}{\Gamma(\rho)}\left(\int_{0}^{t}(t-\eta)^{\frac{\rho-1}{1-\nabla}} d \eta\right)^{1-\nabla}\left(\int_{0}^{t} G_{2}^{1 / \nabla}(\eta) d \eta\right)^{\nabla} \\
\leq & \|\mathfrak{X}(0)\|+\frac{v_{1}\left(\left\|G_{1}\right\|_{1 / \nabla}+\left\|G_{2}\right\|_{1 / \nabla}\right)}{\Gamma(\rho)} T^{M_{1}} \leq \chi_{1} .
\end{aligned}
$$

Similarly, we have

$$
\|\mathcal{X}(t)\| \leq\|\mathcal{X}(0)\|+\frac{v_{2}\left(\left\|G_{3}\right\|_{1 / \nabla}+\left\|G_{4}\right\|_{1 / \nabla}\right)}{\Gamma\left(\rho_{2}\right)} T^{M_{2}} \leq \chi_{2}
$$


and

$$
\|\boldsymbol{j}(t)\| \leq\|\mathbf{3}\|+\frac{v_{3}\left(\left\|G_{5}\right\|_{1 / \nabla}+\left\|G_{6}\right\|_{1 / \nabla}\right)}{\Gamma\left(\rho_{2}\right)} T^{M_{2}} \leq \chi_{3},
$$

and therefore $\mathfrak{X}\left(\Xi_{\chi}\right), \mathfrak{X}\left(\Xi_{\chi}\right), \mathfrak{z}\left(\Xi_{\chi}\right) \in \Xi_{\chi}$.

II) Let us show that $\mathfrak{X}^{*}, \mathfrak{X}^{*}$, and $\mathfrak{Z}^{*}$ are contractions. For $m, z \in \Xi_{\chi}$, we conclude

$$
\begin{array}{rl}
\| \mathfrak{X}^{*} & m(t)-\mathfrak{X}^{* *} z(t) \| \\
\leq & \frac{1}{\Gamma(\rho)} \int_{0}^{t}(t-\eta)^{\rho-1} L(\eta)|m(\eta)-z(\eta)| d \eta \\
\quad \leq & \frac{1}{\Gamma(\rho)}\left(\int_{0}^{t}(t-\eta)^{\frac{\rho-1}{1-\nabla}} d \eta\right)^{1-\nabla}\left(L^{1 / \nabla}(\eta) d \eta\right)^{\nabla}\|m-z\| \leq \Psi_{i}\|m-z\|, \\
\left\|\mathcal{Q}^{*} m(t)-\mathcal{X}^{* *} z(t)\right\| & \\
\quad \leq \frac{1}{\Gamma(\rho)} \int_{0}^{t}(t-\eta)^{\rho-1} L(\eta)|m(\eta)-z(\eta)| d \eta \\
\quad \leq \frac{1}{\Gamma(\rho)}\left(\int_{0}^{t}(t-\eta)^{\frac{\rho-1}{1-\nabla}} d \eta\right)^{1-\nabla}\left(L^{1 / \nabla}(\eta) d \eta\right)^{\nabla}\|m-z\| \leq \Psi_{i}\|m-z\|, \\
\left\|\mathfrak{J}^{*} m(t)-\mathfrak{3}^{* *} z(t)\right\| \\
\quad \leq \frac{1}{\Gamma(\rho)} \int_{0}^{t}(t-\eta)^{\rho-1} L(\eta)|m(\eta)-z(\eta)| d \eta \\
\quad \leq \frac{1}{\Gamma(\rho)}\left(\int_{0}^{t}(t-\eta)^{\frac{\rho-1}{1-\nabla}} d \eta\right)^{1-\nabla}\left(L^{1 / \nabla}(\eta) d \eta\right)^{\nabla}\|m-z\| \leq \Psi_{i}\|m-z\|,
\end{array}
$$

where

$$
\Psi_{i}=\frac{\sigma_{i}\|L\|_{1 / \nabla} T^{\mu_{i}}}{\Gamma(\rho)}<1, \quad 1 \leq i \leq 3
$$

These statements confirm that $\mathfrak{X}^{*}, \mathfrak{Q}^{*}$, and $\mathfrak{Z}^{*}$ are contractions.

III) Let us show that $\mathfrak{X}^{* *}, \mathcal{Z}^{* *}$, and $\mathfrak{Z}^{* *}$ are compact. For $0 \leq j_{1} \leq j_{2} \leq T$, we have

$$
\begin{aligned}
&\left\|\mathfrak{X}^{* *} \varkappa\left(j_{1}\right)-\mathfrak{X}^{* *} z\left(j_{2}\right)\right\| \\
& \leq \leq \frac{1}{\Gamma(\rho)}\left|\int_{0}^{j_{2}}\left(j_{2}-\eta\right)^{\rho-1} \mathfrak{X}^{* *}(\eta, m(\eta)) d \eta-\int_{0}^{j_{1}}\left(j_{1}-\eta\right)^{\rho-1} \mathfrak{X}^{* *}(\eta, m(\eta)) d \eta\right| \\
& \leq \leq \frac{1}{\Gamma(\rho)} \int_{0}^{j_{1}}\left(\left(j_{1}-\eta\right)^{\rho-1}-\left(j_{2}-\eta\right)^{\rho-1}\right) G_{11}(\eta) d \eta+\int_{j_{1}}^{j_{2}}\left(j_{2}-\eta\right)^{\rho-1} G_{11}(\eta) d \eta \\
& \leq \frac{1}{\Gamma(\rho)}\left[\int_{0}^{j_{1}}\left(\left(j_{1}-\eta\right)^{\rho-1}-\left(j_{2}-\eta\right)^{\rho-1}\right)^{\frac{1}{1-\nabla}} d \eta\right]^{1-\nabla}\left(G_{11}^{1 / \nabla}(\eta) d \eta\right)^{\nabla} \\
& \quad+\frac{1}{\Gamma(\rho)} \int_{j_{1}}^{j_{2}}\left(\left(j_{2}-\eta\right)^{\frac{\rho-1}{1-\nabla}} d \eta\right)^{1-\nabla}\left(G_{11}^{1 / \nabla}(\eta) d \eta\right)^{\nabla} \\
& \leq \frac{\sigma_{i}}{\Gamma(\rho)}\left[j_{1}^{\frac{\rho-\nabla}{1-\nabla}}-j 2^{\frac{\rho-\nabla}{1-\nabla}}+\left(j_{2}-j_{1}\right)^{\frac{\rho-\nabla}{1-\nabla}}\right]^{1-\nabla}\left\|G_{11}\right\|_{1 / \nabla}+\frac{\sigma_{i}}{\Gamma(\rho)}\left(j_{2}-j_{1}\right)^{\rho-\nabla}\left\|G_{11}\right\|_{1 / \nabla}
\end{aligned}
$$




$$
\begin{aligned}
& \leq \frac{\sigma_{i}}{\Gamma(\rho)}\left[\left(j_{2}-j_{1}\right)^{\frac{\rho-\nabla}{1-\nabla}}\right]^{1-\nabla}\left\|G_{11}\right\|_{1 / \nabla}+\frac{\sigma_{i}}{\Gamma(\rho)}\left(j_{2}-j_{1}\right)^{\rho-\nabla}\left\|G_{11}\right\|_{1 / \nabla} \\
& \leq \frac{2 \sigma_{i}\left\|G_{11}\right\|_{1 / \nabla}}{\Gamma(\rho)}\left(j_{2}-j_{1}\right)^{\rho-\nabla} .
\end{aligned}
$$

Similarly, we get

$$
\begin{aligned}
\| \mathfrak{X}^{* *} & \varkappa\left(j_{1}\right)-\mathfrak{X}^{* *} z\left(j_{2}\right) \| \\
& \leq \frac{\sigma_{i}}{\Gamma(\rho)}\left[\left(j_{2}-j_{1}\right)^{\frac{\rho-\nabla}{1-\nabla}}\right]^{1-\nabla}\left\|G_{21}\right\|_{1 / \nabla}+\frac{\sigma_{i}}{\Gamma(\rho)}\left(j_{2}-j_{1}\right)^{\rho-\nabla}\left\|G_{21}\right\|_{1 / \nabla} \\
& \leq \frac{2 \sigma_{i}\left\|G_{21}\right\|_{1 / \nabla}}{\Gamma(\rho)}\left(j_{2}-j_{1}\right)^{\rho-\nabla} .
\end{aligned}
$$

Finally, we have

$$
\begin{aligned}
\left\|\boldsymbol{3}^{* *} \varkappa\left(j_{1}\right)-\mathbf{3}^{* *} z\left(j_{2}\right)\right\| & \leq \frac{\sigma_{i}}{\Gamma(\rho)}\left[\left(j_{2}-j_{1}\right)^{\frac{\rho-\nabla}{1-\nabla}}\right]^{1-\nabla}\left\|G_{31}\right\|_{1 / \nabla}+\frac{\sigma_{i}}{\Gamma(\rho)}\left(j_{2}-j_{1}\right)^{\rho-\nabla}\left\|G_{31}\right\|_{1 / \nabla} \\
& \leq \frac{2 \sigma_{i}\left\|G_{31}\right\|_{1 / \nabla}}{\Gamma(\rho)}\left(j_{2}-j_{1}\right)^{\rho-\nabla}
\end{aligned}
$$

for $\sigma_{i}, 1 \leq i \leq 3$.

Now by the Arzelà-Ascoli theorem [15] $\mathfrak{X}^{* *}\left(\Xi_{\xi}\right), \mathfrak{X}^{* *}$, and $\mathfrak{Z}^{* *}$ are relatively compact. So $\mathfrak{X}^{* *}, \mathfrak{X}^{* *}$, and $\mathfrak{\Xi}^{* *}$ are compact.

Since $\mathfrak{X}^{*}, \mathfrak{X}^{*}, \mathfrak{Z}^{*}$, and $\mathfrak{w}^{*}$ are contractions and $\mathfrak{X}^{* *}, \mathfrak{X}^{* *}$ and $\mathfrak{Z}^{* *}$ are compact and therefore continuous, the maps $\mathfrak{X}=\mathfrak{X}^{*}+\mathfrak{X}^{* *}, \mathfrak{X}=\mathfrak{X}^{*}+\mathfrak{X}^{* *}$, and $\mathfrak{Z}=\mathfrak{Z}^{*}+\mathfrak{Z}^{* *}$ are condensing on $\Xi_{\xi}$. Hence the existence of a fixed point for each point $\mathfrak{X}, \mathfrak{X}$, and $\mathfrak{Z}$ is proved.

IV) We will show that the problem has a unique solution. To this end, let us define the map $H$ as follows:

$$
\begin{aligned}
& H[\mathfrak{X}(t)]=\mathfrak{X}(0)+\frac{1}{\Gamma(\rho)} \int_{0}^{t}(t-\eta)^{\rho-1} \mathfrak{X}(\eta, \mathfrak{X}(\eta)) d \eta, \\
& H[\mathfrak{X}(t)]=\mathfrak{X}(0)+\frac{1}{\Gamma(\rho)} \int_{0}^{t}(t-\eta)^{\rho-1} \mathfrak{X}(\eta, \mathfrak{X}(\eta)) d \eta, \\
& H[\mathfrak{z}(t)]=\mathfrak{Z} h(0)+\frac{1}{\Gamma(\rho)} \int_{0}^{t}(t-\eta)^{\rho-1} \mathfrak{z}(\eta, \mathfrak{z}(\eta)) d \eta .
\end{aligned}
$$

For $\mathfrak{X}^{*}(t), \mathfrak{X}^{* *}(t), \mathfrak{X}^{*}(t), \mathfrak{X}^{* *}(t), \mathfrak{Z}^{*}(t), \mathfrak{Z}^{* *}(t)$, we obtain

$$
\begin{aligned}
\left|H\left[\mathfrak{X}^{*}(t)\right]-F\left[\mathfrak{X}^{* *}(t)\right]\right| & \leq \frac{1}{\Gamma(\rho)} \int_{0}^{t}(t-\eta)^{\rho-1} L_{1}(\eta)\left|\mathfrak{X}^{*}(\eta)-\mathfrak{X}^{* *}(\eta)\right| d \eta \\
& \leq \frac{1}{\Gamma(\rho)}\left[\int_{0}^{t}(t-\eta)^{\frac{\rho-1}{1-\nabla}} d \eta\right]^{1-\nabla}\left[\int_{0}^{t} L_{1}^{1 / \nabla}(\eta) d \eta\right]^{\nabla} \\
& \leq \frac{\sigma_{1}\left\|L_{1}\right\|_{1 / \nabla} T^{M_{1}}}{\Gamma(\rho)}\left\|\mathfrak{X}^{*}-\mathfrak{X}^{* *}\right\| .
\end{aligned}
$$

Besides, we have

$$
\left|H\left[\mathfrak{X}^{*}(t)\right]-H\left[\mathfrak{X}^{* *}(t)\right]\right| \leq \frac{\sigma_{2}\left\|L_{2}\right\|_{1 / \nabla} T^{M_{2}}}{\Gamma(\rho)}\left\|\mathfrak{X}^{*}-\mathfrak{X}^{* *}\right\|
$$


and

$$
\left|H\left[\boldsymbol{J}^{*}(t)\right]-H\left[\boldsymbol{\Xi}^{* *}(t)\right]\right| \leq \frac{\sigma_{3}\left\|L_{3}\right\|_{1 / \nabla} T^{M_{3}}}{\Gamma(\rho)}\left\|\boldsymbol{3}^{*}-\boldsymbol{\Xi}^{* *}\right\| .
$$

These results indicate that model (7) will always has a unique solution.

\subsection{The numerical method}

In this section, we use the Adams-Bashforth-Moulton (ABM) numerical method. Here we follow the steps to apply this method in solving the following fractional-order problem:

$$
{ }_{0}^{C} \mathcal{D}_{t}^{\alpha} \phi(t)=\Xi(t, \phi(t)), \quad \phi^{k}(0)=\phi_{0}^{k}, k=0,1, \ldots, n-1 .
$$

Now taking the Liouville-Caputo fractional integration on (24) yields

$$
\phi(t)=\sum_{k=0}^{n-1} f_{0}^{(k)} \frac{t^{k}}{k !}+\frac{1}{\Gamma(\boldsymbol{\alpha})} \int_{0}^{t}(t-\eta)^{\boldsymbol{\alpha}-1} \Xi(\eta, \phi(\eta)) d \eta .
$$

The following predictor-corrector form determines an approximate solution to the problem [31]:

$$
\begin{aligned}
\phi_{k+1}^{P} & =\sum_{\zeta=0}^{n-1} \frac{t_{k+1}^{\zeta}}{\zeta !} \phi_{0}^{(\zeta)}+\frac{1}{\Gamma(\boldsymbol{\alpha})} \sum_{\zeta=0}^{k} \theta_{\zeta, k+1} \Xi\left(t_{\zeta}, f_{\zeta}\right), \\
\phi_{k+1} & =\sum_{\zeta=0}^{n-1} \frac{t_{k+1}^{\zeta}}{\zeta !} \phi_{0}^{(\zeta)}+\frac{1}{\Gamma(\boldsymbol{\alpha})}\left(\sum_{\zeta=0}^{k} \gamma_{\zeta, k+1} \Xi\left(t_{\zeta}, f_{\zeta}\right)+\gamma_{k+1, k+1} \Xi\left(t_{k+1}, \phi_{k+1}^{P}\right)\right),
\end{aligned}
$$

where

$$
\begin{array}{ll}
\gamma_{\zeta, k+1}=\frac{\hbar^{\alpha}}{\alpha(\boldsymbol{\alpha}+1)} \cdot \begin{cases}k^{\alpha+1}-(k-\alpha)(k+1)^{\alpha}, & \zeta=0, \\
(-\zeta+k+2)^{\alpha+1}+(-\zeta+k)^{\alpha+1}-2(-\zeta+k+1)^{\alpha+1}, & 1 \leq \zeta \leq k, \\
1, & \zeta=k+1,\end{cases} \\
\theta_{\zeta, k+1}=\frac{\hbar^{\alpha}}{\alpha}\left((-\zeta+k+1)^{\alpha}-(-\zeta+k)^{\alpha}\right), & \zeta=0,1,2, \ldots, k .
\end{array}
$$

Utilizing the numerical algorithm presented in (26), we determine an approximate solution to the fractional problem (7) from the formulae

$$
\begin{aligned}
\mathcal{T}(t)= & \sum_{k=0}^{n-1} \mathcal{T}^{(k)}(0) \frac{t^{k}}{k !} \\
& +\frac{1}{\Gamma(\boldsymbol{\alpha})} \int_{0}^{t}(t-\eta)^{\boldsymbol{\alpha}-1}\left[\mathcal{T}(\eta)(1-\mathcal{T}(\eta))-\beta_{12} \mathcal{T}(\eta) \mathcal{H}(\eta)-\beta_{13} \mathcal{T}(\eta) \mathcal{E}(\eta)\right] d \eta \\
\mathcal{H}(t)= & \sum_{k=0}^{n-1} \mathcal{H}^{(k)}(0) \frac{t^{k}}{k !}+\frac{1}{\Gamma(\boldsymbol{\alpha})} \int_{0}^{t}(t-\eta)^{\boldsymbol{\alpha}-1}\left[k_{2} \mathcal{H}(\eta)(1-\mathcal{H}(\eta))-\beta_{21} \mathcal{T}(\eta) \mathcal{H}(\eta)\right] d \eta \\
\mathcal{E}(t)= & \sum_{k=0}^{n-1} \mathcal{E}^{(k)}(0) \frac{t^{k}}{k !}+\frac{1}{\Gamma(\boldsymbol{\alpha})} \int_{0}^{t}(t-\eta)^{\boldsymbol{\alpha}-1}\left[\frac{k_{3} \mathcal{T}(\eta) \mathcal{E}(\eta)}{\mathcal{T}(\eta)+s_{3}}-\beta_{31} \mathcal{T}(\eta) \mathcal{E}(\eta)-c_{3} \mathcal{E}(\eta)\right] d \eta
\end{aligned}
$$




\subsection{Numerical simulations}

Figures 1-3 demonstrate the variation of state variables in model (7) when the scheme (26) is utilized for different values of $\alpha \in(0,1]$. In this simulations, we have considered the following values in the model: $\beta_{12}=1, \beta_{13}=2.5, k_{2}=0.6, \beta_{21}=1.5, k_{3}=4.5, s_{3}=1, a_{31}=0.2$, and $d_{3}=0.5$. In our performed numerical simulations, we take $t_{\text {final }}=500$ and $\hbar=0.001$. In Fig. 1 , we take $\left.(\mathcal{T}(t), \mathcal{H}(t), \mathcal{E}(t))\right|_{t=0}=(0.1,0.1,0.1)$. In this case the model exhibits chaotic attractor behavior. Also, by taking into consideration $\left.(\mathcal{T}(t), \mathcal{H}(t), \mathcal{E}(t))\right|_{t=0}=(0.3,0.3,0.3)$, and $\beta_{12}=0.745$ the model shows the limit cycle behavior in Fig. 2, whereas for $\mathcal{T}(0)=$ $0.3517, \mathcal{H}(0)=0.1115, \mathcal{E}(0)=0.4951$, and $\beta_{12}=0.920$, we get periodic orbit trajectories in the solutions as depicted in Fig. 3.
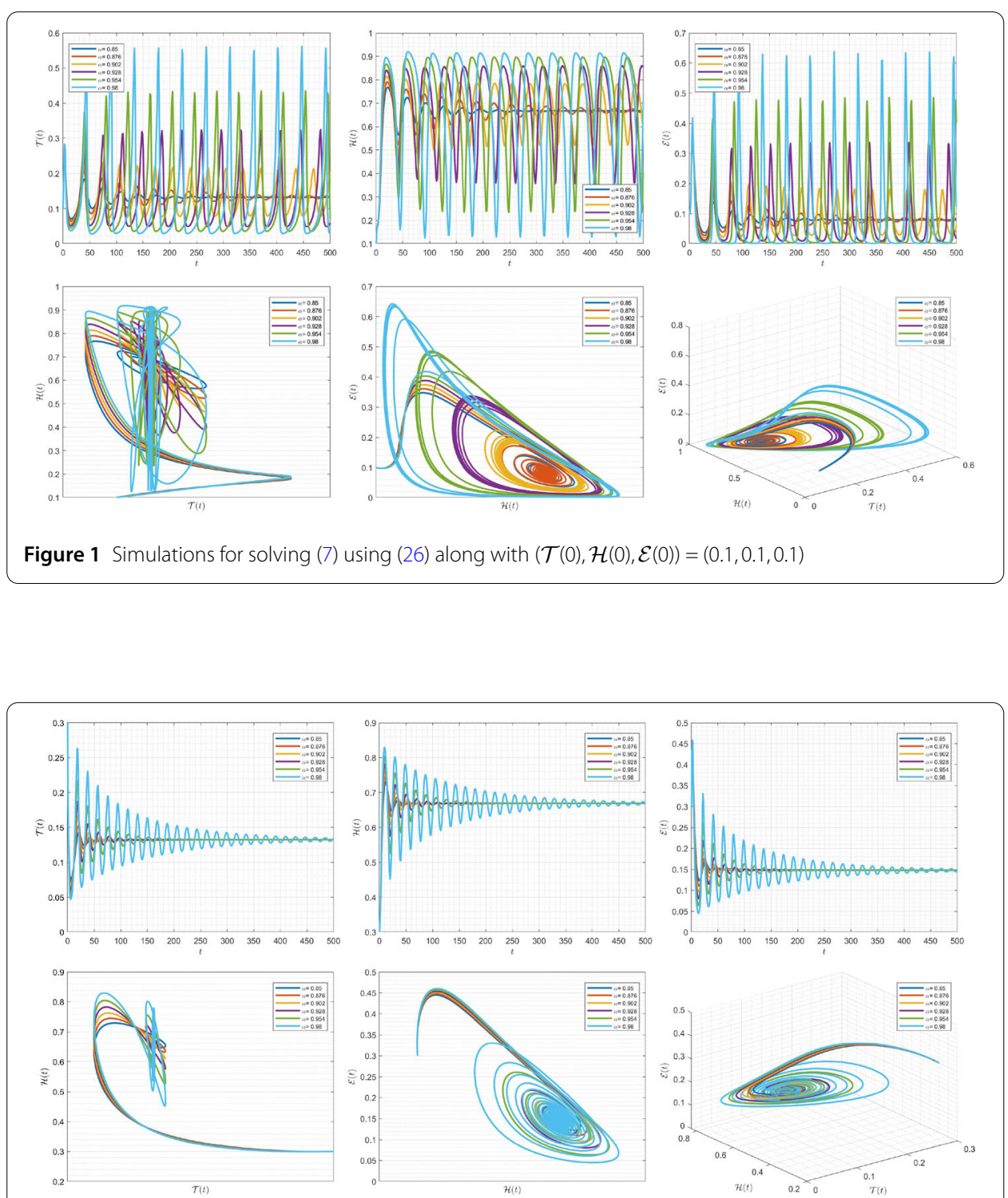

Figure 2 Simulations for solving (7) using (26) along with $(\mathcal{T}(0), \mathcal{H}(0), \mathcal{E}(0))=(0.3,0.3,0.3)$ and $\beta_{12}=0.745$ 

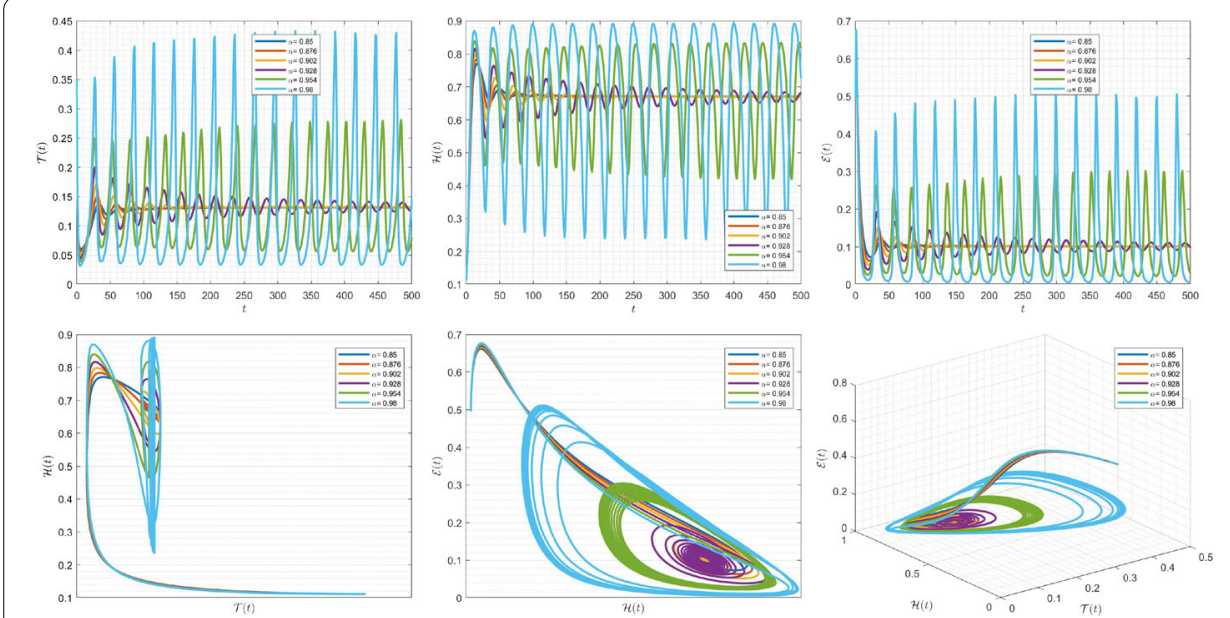

Figure 3 Simulations for solving (7) using (26) along with $(\mathcal{T}(0), \mathcal{H}(0), \mathcal{E}(0))=(0.3517,0.1115,0.4951)$ and $\beta_{12}=0.92$

\section{The model via Caputo-Fabrizio-Caputo fractional derivative}

In this section, we study the following model:

$$
\begin{aligned}
& { }_{0}^{\mathrm{CFC}} \mathcal{D}_{t}^{\alpha} \mathcal{T}(t)=\mathcal{T}(t)(1-\mathcal{T}(t))-\beta_{12} \mathcal{T}(t) \mathcal{H}(t)-\beta_{13} \mathcal{T}(t) \mathcal{E}(t), \\
& { }_{0}^{\mathrm{CFC}} \mathcal{D}_{t}^{\alpha} \mathcal{H}(t)=k_{2} \mathcal{H}(t)(1-\mathcal{H}(t))-\beta_{21} \mathcal{T}(t) \mathcal{H}(t), \\
& { }_{0}^{\mathrm{CFC}} \mathcal{D}_{t}^{\alpha} \mathcal{E}(t)=\frac{k_{3} \mathcal{T}(t) \mathcal{E}(t)}{\mathcal{T}(t)+s_{3}}-\beta_{31} \mathcal{T}(t) \mathcal{E}(t)-c_{3} \mathcal{E}(t) .
\end{aligned}
$$

The fractional derivative operator ${ }_{0}^{\mathrm{CFC}} \mathcal{D}_{t}^{\alpha}$ in this model is Caputo-Fabrizio-Caputo (CFC) given by [13]

$$
{ }_{0}^{\mathrm{CFC}} \mathcal{D}_{t}^{\alpha} \phi(t)=\frac{\mathrm{M}(\boldsymbol{\alpha})}{n-\boldsymbol{\alpha}} \int_{0}^{t} \phi^{\prime}(\eta) \exp \left[-\frac{\boldsymbol{\alpha}}{1-\boldsymbol{\alpha}}(t-\eta)\right] d \eta, \quad 0<\boldsymbol{\alpha} \leq 1,
$$

where

$$
M(\boldsymbol{\alpha})=\frac{2}{2-\boldsymbol{\alpha}}, \quad 0<\boldsymbol{\alpha}<1 .
$$

The CFC fractional integral is also defined by [33]

$$
{ }_{0}^{\mathrm{CFC}} \mathcal{I}_{t}^{\boldsymbol{\alpha}} \phi(t)=\frac{2(1-\boldsymbol{\alpha})}{(2-\boldsymbol{\alpha}) \mathrm{M}(\boldsymbol{\alpha})} \phi(t)+\frac{2 \boldsymbol{\alpha}}{(2-\boldsymbol{\alpha}) \mathrm{M}(\boldsymbol{\alpha})} \int_{0}^{t} \phi(\eta) d \eta, \quad t \geq 0
$$

\subsection{Existence of the coupled solutions}

Applying the CFC integral definition (29), we obtain the following relationships:

$$
\left\{\begin{array}{l}
\mathcal{T}(t)-\mathcal{T}(0)={ }_{0}^{\mathrm{CFC}} \mathcal{I}_{t}^{\alpha}\left\{\mathcal{T}(t)(1-\mathcal{T}(t))-\beta_{12} \mathcal{T}(t) \mathcal{H}(t)-\beta_{13} \mathcal{T}(t) \mathcal{E}(t)\right\} \\
\mathcal{H}(t)-\mathcal{H}(0)={ }_{0}^{\mathrm{CFC}} \mathcal{I}_{t}^{\alpha}\left\{k_{2} \mathcal{H}(t)(1-\mathcal{H}(t))-\beta_{21} \mathcal{T}(t) \mathcal{H}(t)\right\} \\
\mathcal{E}(t)-\mathcal{E}(0)={ }_{0}^{\mathrm{CFC}} \mathcal{I}_{t}^{\alpha}\left\{\frac{k_{3} \mathcal{T}(t) \mathcal{E}(t)}{\mathcal{T}(t)+s_{3}}-\beta_{31} \mathcal{T}(t) \mathcal{E}(t)-c_{3} \mathcal{E}(t)\right\}
\end{array}\right.
$$


Now we consider the following kernels:

$$
\left\{\begin{array}{l}
\mathcal{K}_{1}(t, \mathcal{T}(t), \mathcal{H}(t), \mathcal{E}(t))=\mathcal{T}(t)(1-\mathcal{T}(t))-\beta_{12} \mathcal{T}(t) \mathcal{H}(t)-\beta_{13} \mathcal{T}(t) \mathcal{E}(t) \\
\mathcal{K}_{2}(t, \mathcal{T}(t), \mathcal{H}(t), \mathcal{E}(t))=k_{2} \mathcal{H}(t)(1-\mathcal{H}(t))-\beta_{21} \mathcal{T}(t) \mathcal{H}(t) \\
\mathcal{K}_{3}(t, \mathcal{T}(t), \mathcal{H}(t), \mathcal{E}(t))=\frac{k_{3} \mathcal{T}(t) \mathcal{E}(t)}{\mathcal{T}(t)+s_{3}}-\beta_{31} \mathcal{T}(t) \mathcal{E}(t)-c_{3} \mathcal{E}(t)
\end{array}\right.
$$

Theorem 2 The initial value problem and the kernels $\mathcal{K}_{1}(\mathcal{T}(t), \mathcal{H}(t), \mathcal{E}(t)), \mathcal{K}_{2}(\mathcal{T}(t), \mathcal{H}(t)$, $\mathcal{E}(t))$, and $\mathcal{K}_{3}(\mathcal{T}(t), \mathcal{H}(t), \mathcal{E}(t))$ satisfy the Lipschitz condition.

Proof See [24].

Theorem 3 The fractional nonlinear system (29) admits at least one solution.

Proof See [24].

Theorem 4 The fractional nonlinear system (29) always admits a unique solution.

Proof See [24].

\subsection{Numerical method}

Now let us focus on determining an approximate solution to the following CFC fractional Cauchy problem:

$$
{ }_{0}^{\mathrm{CFC}} \mathcal{D}_{t}^{\alpha} \phi(t)=\Xi(t, \phi(t))
$$

Using the corresponding fractional integral operator yields

$$
\phi(t)-\phi(0)=\frac{1-\boldsymbol{\alpha}}{\mathrm{M}(\boldsymbol{\alpha})} \Xi(t, \phi(t))+\frac{\boldsymbol{\alpha}}{\mathrm{M}(\boldsymbol{\alpha})} \int_{0}^{t} \Xi(\eta, \phi(\eta)) d \eta .
$$

Taking $t=t_{n+1}$ in (36), we have

$$
\phi\left(t_{n+1}\right)-\phi(0)=\frac{(2-\boldsymbol{\alpha})(1-\boldsymbol{\alpha})}{2} \Xi\left(t_{n}, \phi\left(t_{n}\right)\right)+\frac{\boldsymbol{\alpha}(2-\boldsymbol{\alpha})}{2} \int_{0}^{t_{n+1}} \Xi(\eta, \phi(\eta)) d \eta
$$

and

$$
\phi\left(t_{n}\right)-\phi(0)=\frac{(2-\boldsymbol{\alpha})(1-\boldsymbol{\alpha})}{2} \Xi\left(t_{n-1}, \phi\left(t_{n-1}\right)\right)+\frac{\boldsymbol{\alpha}(2-\boldsymbol{\alpha})}{2} \int_{0}^{t_{n}} \Xi(\eta, \phi(\eta)) d \eta
$$

Inserting Eq. (38) into Eq. (37), we get

$$
\begin{aligned}
\phi\left(t_{n+1}\right)= & \phi\left(t_{n}\right)+\frac{(2-\boldsymbol{\alpha})(1-\boldsymbol{\alpha})}{2}\left[\Xi\left(t_{n}, \phi\left(t_{n}\right)\right)-\Xi\left(t_{n-1}, \phi\left(t_{n-1}\right)\right)\right] \\
& +\frac{\boldsymbol{\alpha}(2-\boldsymbol{\alpha})}{2} \int_{t_{n}}^{t_{n+1}} \Xi(\eta, \phi(\eta)) d \eta,
\end{aligned}
$$

where

$$
\int_{t_{n}}^{t_{n+1}} \Xi(\eta, \phi(\eta)) d \eta=\frac{3 \hbar}{2} \Xi\left(t_{n}, f_{n}\right)-\frac{\hbar}{2} \Xi\left(t_{n-1}, f_{n-1}\right) .
$$


So we have

$$
\begin{aligned}
f_{n+1}= & f_{n}+\left[\frac{(2-\boldsymbol{\alpha})(1-\boldsymbol{\alpha})}{2}+\frac{3 \hbar}{4} \boldsymbol{\alpha}(2-\boldsymbol{\alpha})\right] \Xi\left(t_{n}, f_{n}\right) \\
& -\left[\frac{(2-\boldsymbol{\alpha})(1-\boldsymbol{\alpha})}{2}+\frac{\hbar}{4} \boldsymbol{\alpha}(2-\boldsymbol{\alpha})\right] \Xi\left(t_{n-1}, f_{n-1}\right) .
\end{aligned}
$$

As a result, the following recursive relations are determined to approximate the CFC problem (29) as in [22]:

$$
\begin{aligned}
\mathcal{T}_{n+1}(t)= & \mathcal{T}(0)+\left[\frac{(2-\boldsymbol{\alpha})(1-\boldsymbol{\alpha})}{2}+\frac{3 \hbar}{4} \boldsymbol{\alpha}(2-\boldsymbol{\alpha})\right] \Xi_{1}\left(\mathcal{T}_{n}(t), \mathcal{H}_{n}(t), \mathcal{E}_{n}(t), t_{n}\right) \\
& -\left[\frac{(2-\boldsymbol{\alpha})(1-\boldsymbol{\alpha})}{2}+\frac{\hbar}{4} \boldsymbol{\alpha}(2-\boldsymbol{\alpha})\right] \Xi_{1}\left(\mathcal{T}_{n}(t), \mathcal{H}_{n}(t), \mathcal{E}_{n}(t), t_{n}\right), \\
\mathcal{H}_{n+1}(t)= & \mathcal{H}(0)+\left[\frac{(2-\boldsymbol{\alpha})(1-\boldsymbol{\alpha})}{2}+\frac{3 \hbar}{4} \boldsymbol{\alpha}(2-\boldsymbol{\alpha})\right] \Xi_{2}\left(\mathcal{T}_{n}(t), \mathcal{H}_{n}(t), \mathcal{E}_{n}(t), t_{n}\right) \\
& -\left[\frac{(2-\boldsymbol{\alpha})(1-\boldsymbol{\alpha})}{2}+\frac{\hbar}{4} \boldsymbol{\alpha}(2-\boldsymbol{\alpha})\right] \Xi_{2}\left(\mathcal{T}_{n}(t), \mathcal{H}_{n}(t), \mathcal{E}_{n}(t), t_{n}\right), \\
\mathcal{E}_{n+1}(t)= & \mathcal{E}(0)+\left[\frac{(2-\boldsymbol{\alpha})(1-\boldsymbol{\alpha})}{2}+\frac{3 \hbar}{4} \boldsymbol{\alpha}(2-\boldsymbol{\alpha})\right] \Xi_{3}\left(\mathcal{T}_{n}(t), \mathcal{H}_{n}(t), \mathcal{E}_{n}(t), t_{n}\right) \\
& -\left[\frac{(2-\boldsymbol{\alpha})(1-\boldsymbol{\alpha})}{2}+\frac{\hbar}{4} \boldsymbol{\alpha}(2-\boldsymbol{\alpha})\right] \Xi_{3}\left(\mathcal{T}_{n}(t), \mathcal{H}_{n}(t), \mathcal{E}_{n}(t), t_{n}\right),
\end{aligned}
$$

where $\Xi_{1}=\mathcal{T}(t)(1-\mathcal{T}(t))-\beta_{12} \mathcal{T}(t) \mathcal{H}(t)-\beta_{13} \mathcal{T}(t) \mathcal{E}(t), \quad \Xi_{2}=k_{2} \mathcal{H}(t)(1-\mathcal{H}(t))-$ $\beta_{21} \mathcal{T}(t) \mathcal{H}(t)$, and $\Xi_{3}=\frac{k_{3} \mathcal{T}(t) \mathcal{E}(t)}{\mathcal{T}(t)+s_{3}}-a_{31} \mathcal{T}(t) \mathcal{E}(t)-c_{3} \mathcal{E}(t)$.

\subsection{Numerical simulations}

Figures 4-6 are plotted to demonstrate the variation of state variables in model (29) when the scheme (42) is employed for different values of $\alpha \in(0,1]$. In these simulations, we considered the following values in the model: $\beta_{12}=1, \beta_{13}=2.5, k_{2}=0.6, \beta_{21}=1.5, k_{3}=4.5$,

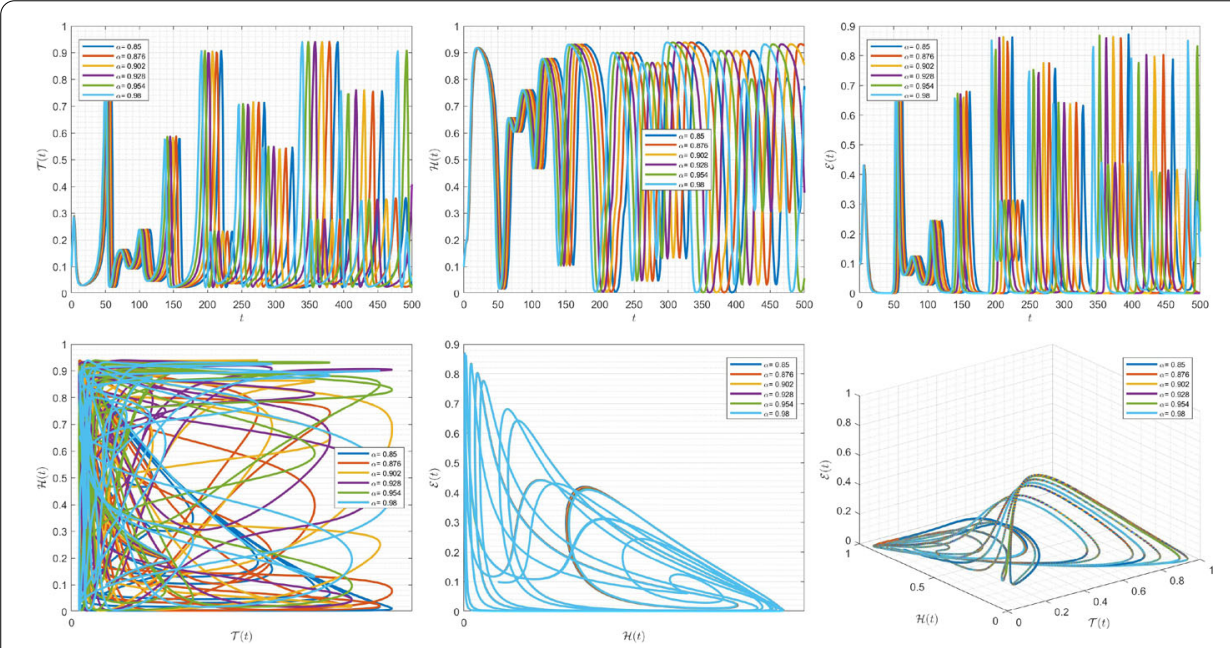

Figure 4 Simulations for solving (29) using (42) along with $(\mathcal{T}(0), \mathcal{H}(0), \mathcal{E}(0))=(0.1,0.1,0.1)$ 

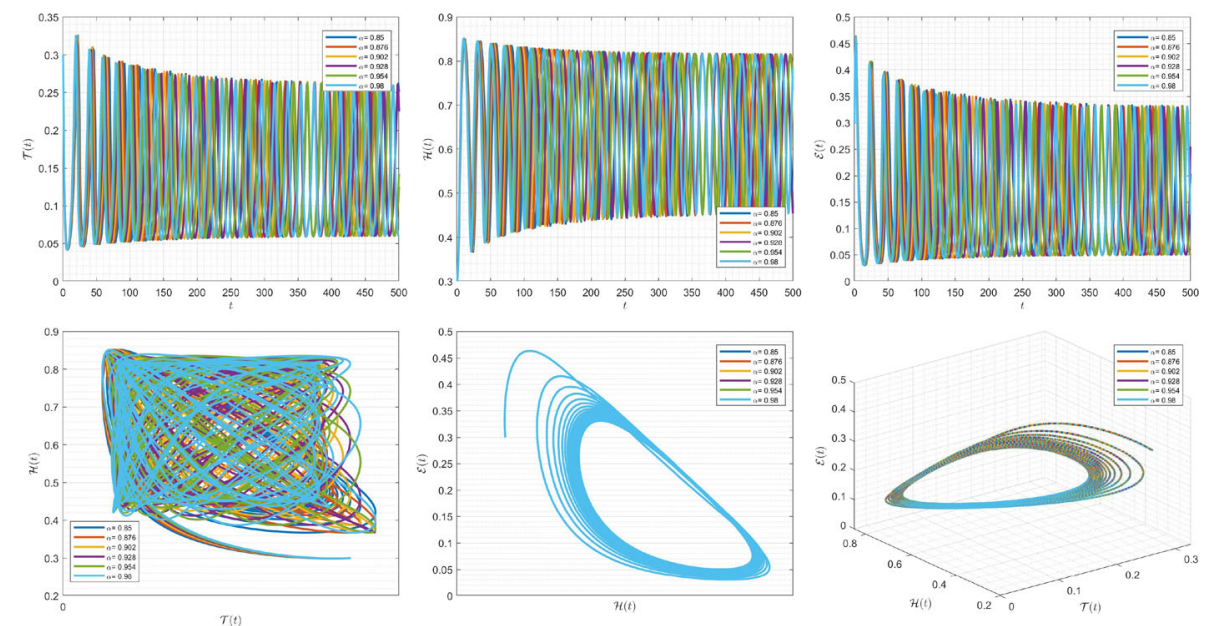

Figure 5 Simulations for solving (29) using (42) along with $(\mathcal{T}(0), \mathcal{H}(0), \mathcal{E}(0))=(0.3,0.3,0.3)$ and $\beta_{12}=0.745$
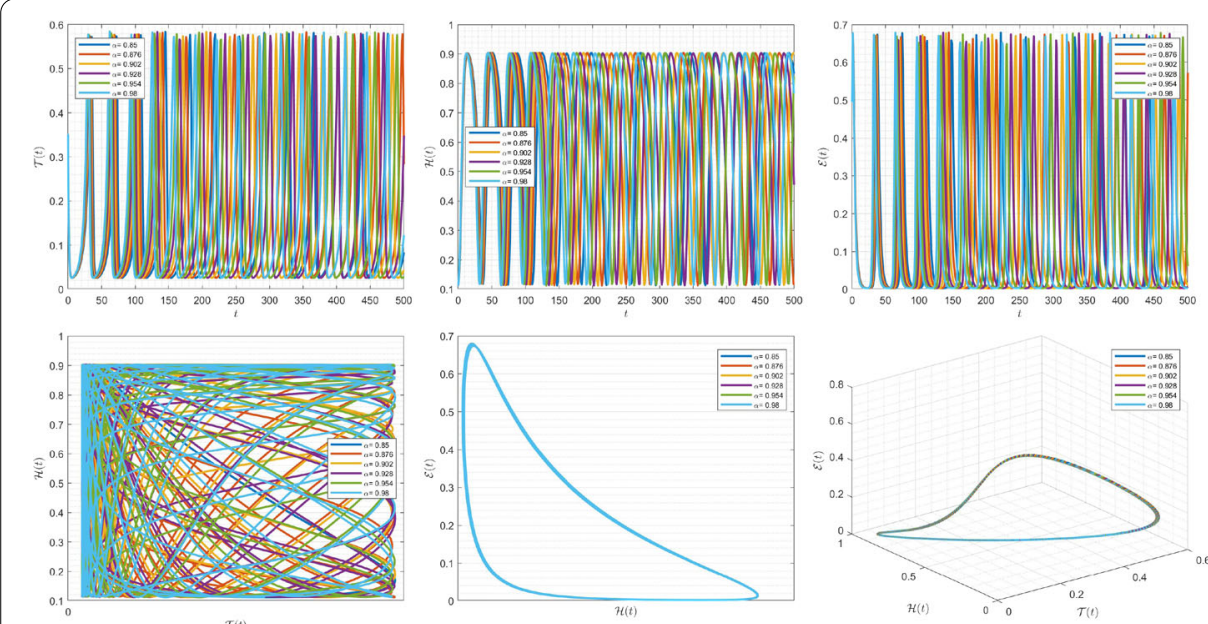

Figure 6 Simulations for solving (29) using (42) along with $(\mathcal{T}(0), \mathcal{H}(0), \mathcal{E}(0))=(0.3517,0.1115,0.4951)$ and $\beta_{12}=0.92$

$s_{3}=1, a_{31}=0.2$, and $d_{3}=0.5$. In our performed numerical simulations, we considered $t_{\text {final }}=500$ and $\hbar=0.001$. In Fig. 4 , we use $\left.(\mathcal{T}(t), \mathcal{H}(t), \mathcal{E}(t))\right|_{t=0}=(0.1,0.1,0.1)$. In this case the model exhibits chaotic attractor behavior. Also, starting from $\left.(\mathcal{T}(t), \mathcal{H}(t), \mathcal{E}(t))\right|_{t=0}=$ $(0.3,0.3,0.3)$ and $\beta_{12}=0.745$, the model shows the limit cycle behavior in Fig. 5. Further, by taking $\mathcal{T}(0)=0.3517, \mathcal{H}(0)=0.1115, \mathcal{E}(0)=0.4951$, and $\beta_{12}=0.920$, we get periodic orbit trajectories in the solutions as depicted in Fig. 6.

\section{The model via Atangana-Baleanu-Caputo fractional derivative}

Now let us consider the model via the Atangana-Baleanu-Caputo fractional derivative as

$$
\begin{aligned}
& { }_{0}^{\mathrm{ABC}} \mathcal{D}_{t}^{\alpha} \mathcal{T}(t)=\mathcal{T}(t)(1-\mathcal{T}(t))-\beta_{12} \mathcal{T}(t) \mathcal{H}(t)-\beta_{13} \mathcal{T}(t) \mathcal{E}(t), \\
& { }_{0}^{\mathrm{ABC}} \mathcal{D}_{t}^{\alpha} \mathcal{H}(t)=k_{2} \mathcal{H}(t)(1-\mathcal{H}(t))-\beta_{21} \mathcal{T}(t) \mathcal{H}(t),
\end{aligned}
$$




$$
{ }_{0}^{\mathrm{ABC}} \mathcal{D}_{t}^{\alpha} \mathcal{E}(t)=\frac{k_{3} \mathcal{T}(t) \mathcal{E}(t)}{\mathcal{T}(t)+s_{3}}-\beta_{31} \mathcal{T}(t) \mathcal{E}(t)-c_{3} \mathcal{E}(t)
$$

where the Atangana-Baleanu fractional integral of order $\alpha$ of a function $\phi(t)$ is defined as [10]

$$
{ }_{0}^{\mathrm{ABC}} \mathcal{D}_{t}^{\alpha} \phi(t)=\frac{\mathrm{B}(\boldsymbol{\alpha})}{n-\boldsymbol{\alpha}} \int_{0}^{t} \phi^{\prime}(\eta) E_{\boldsymbol{\alpha}}\left[-\frac{\boldsymbol{\alpha}}{n-\boldsymbol{\alpha}}(t-\eta)^{\alpha}\right] d \eta,
$$

where $\mathrm{B}(\boldsymbol{\alpha})=1-\alpha+\frac{\alpha}{\Gamma(\alpha)}$ is a normalization function.

The Atangana-Baleanu fractional integral of order $\alpha$ of a function $\phi(t)$ is also defined as [10]

$$
{ }_{0}^{\mathrm{ABC}} \mathcal{I}_{t}^{\boldsymbol{\alpha}} \phi(t)=\frac{1-\boldsymbol{\alpha}}{\mathrm{B}(\boldsymbol{\alpha})} \phi(t)+\frac{\boldsymbol{\alpha}}{\mathrm{B}(\boldsymbol{\alpha}) \Gamma(\boldsymbol{\alpha})} \int_{0}^{t} \phi(\eta)(t-\eta)^{\boldsymbol{\alpha}-1} d \eta .
$$

Taking the definition of the Atangana-Baleanu fractional integral (45) on both sides of equations in system (43), we get the Volterra integral system

$$
\begin{aligned}
\mathcal{T}(t) & -\mathcal{T}(0) \\
= & \frac{1-\boldsymbol{\alpha}}{\mathrm{B}(\boldsymbol{\alpha})}\left\{\mathcal{T}(t)(1-\mathcal{T}(t))-\beta_{12} \mathcal{T}(t) \mathcal{H}(t)-\beta_{13} \mathcal{T}(t) \mathcal{E}(t)\right\} \\
& +\frac{\boldsymbol{\alpha}}{\mathrm{B}(\boldsymbol{\alpha}) \Gamma(\boldsymbol{\alpha})} \int_{0}^{t}\left\{\mathcal{T}(\eta)(1-\mathcal{T}(\eta))-\beta_{12} \mathcal{T}(\eta) \mathcal{H}(\eta)-\beta_{13} \mathcal{T}(t) \mathcal{E}(t)\right\}(t-\eta)^{\alpha-1} d \eta \\
\mathcal{H}(t) & -\mathcal{H}(0) \\
= & \frac{1-\boldsymbol{\alpha}}{\mathrm{B}(\boldsymbol{\alpha})}\left\{k_{2} \mathcal{H}(t)(1-\mathcal{H}(t))-\beta_{21} \mathcal{T}(t) \mathcal{H}(t)\right\} \\
& +\frac{\boldsymbol{\alpha}}{\mathrm{B}(\boldsymbol{\alpha}) \Gamma(\boldsymbol{\alpha})} \int_{0}^{t}\left\{k_{2} \mathcal{H}(\eta)(1-\mathcal{H}(\eta))-\beta_{21} \mathcal{T}(\eta) \mathcal{H}(\eta)\right\}(t-\eta)^{\alpha-1} d \eta \\
\mathcal{E}(t)- & \mathcal{E}(0) \\
= & \frac{1-\boldsymbol{\alpha}}{\mathrm{B}(\boldsymbol{\alpha})}\left\{\frac{k_{3} \mathcal{T}(t) \mathcal{E}(t)}{\mathcal{T}(t)+s_{3}}-\beta_{31} \mathcal{T}(t) \mathcal{E}(t)-c_{3} \mathcal{E}(t)\right\} \\
& +\frac{\boldsymbol{\alpha}}{\mathrm{B}(\boldsymbol{\alpha}) \Gamma(\boldsymbol{\alpha})} \int_{0}^{t}\left\{\frac{k_{3} \mathcal{T}(\eta) \mathcal{E}(\eta)}{\mathcal{T}(\eta)+s_{3}}-\beta_{31} \mathcal{T}(\eta) \mathcal{E}(\eta)-c_{3} \mathcal{E}(\eta)\right\} d \eta
\end{aligned}
$$

and the following iterative formulas:

$$
\begin{aligned}
& \mathcal{T}_{n+1}(t) \\
& \left.=\mathcal{T}(0)+\frac{1-\boldsymbol{\alpha}}{\mathrm{B}(\boldsymbol{\alpha})}\left\{\mathcal{T}_{n}(t)\left(1-\mathcal{T}_{n}(t)\right)-\beta_{12} \mathcal{T}_{n}(t) \mathcal{H}_{n}(t)-\beta_{13} \mathcal{T}_{n}(t) \mathcal{E}_{n}(t)\right\}\right\} \\
& \quad+\frac{\boldsymbol{\alpha}}{\mathrm{B}(\boldsymbol{\alpha}) \Gamma(\boldsymbol{\alpha})} \int_{0}^{t}\left\{\mathcal{T}_{n}(\eta)\left(1-\mathcal{T}_{n}(\eta)\right)-\beta_{12} \mathcal{T}_{n}(\eta) \mathcal{H}_{n}(\eta)-\beta_{13} \mathcal{T}_{n}(\eta) \mathcal{E}_{n}(\eta)\right\}(t-\eta)^{\boldsymbol{\alpha}-1} d \eta \\
& \mathcal{H}_{n+1}(t) \\
& =\mathcal{H}(0)+\frac{1-\boldsymbol{\alpha}}{\mathrm{B}(\boldsymbol{\alpha})}\left\{k_{2} \mathcal{H}_{n}(t)\left(1-\mathcal{H}_{n}(t)\right)-\beta_{21} \mathcal{T}_{n}(t) \mathcal{H}_{n}(t)\right\} \\
& \quad+\frac{\boldsymbol{\alpha}}{\mathrm{B}(\boldsymbol{\alpha}) \Gamma(\boldsymbol{\alpha})} \int_{0}^{t}\left\{k_{2} \mathcal{H}_{n}(\eta)\left(1-\mathcal{H}_{n}(\eta)\right)-\beta_{21} \mathcal{T}_{n}(\eta) \mathcal{H}_{n}(\eta)\right\}(t-\eta)^{\alpha-1} d \eta
\end{aligned}
$$




$$
\begin{aligned}
\mathcal{E}_{n+1}(t) & \\
= & \mathcal{E}(0)+\frac{1-\boldsymbol{\alpha}}{\mathrm{B}(\boldsymbol{\alpha})}\left\{\frac{k_{3} \mathcal{T}_{n}(t) \mathcal{E}_{n}(t)}{\mathcal{T}_{n}(t)+s_{3}}-\beta_{31} \mathcal{T}_{n}(t) \mathcal{E}_{n}(t)-c_{3} \mathcal{E}_{n}(t)\right\} \\
& +\frac{\boldsymbol{\alpha}}{\mathrm{B}(\boldsymbol{\alpha}) \Gamma(\boldsymbol{\alpha})} \int_{0}^{t}\left\{\frac{k_{3} \mathcal{T}_{n}(\eta) \mathcal{E}_{n}(\eta)}{\mathcal{T}_{n}(\eta)+s_{3}}-\beta_{31} \mathcal{T}_{n}(\eta) \mathcal{E}_{n}(\eta)-c_{3} \mathcal{E}_{n}(\eta)\right\}(t-\eta)^{\boldsymbol{\alpha}-1} d \eta,
\end{aligned}
$$

where

$$
\mathcal{T}_{0}(t)=\mathcal{T}(0), \quad \mathcal{H}_{0}(t)=\mathcal{H}(0), \quad \mathcal{E}_{0}(t)=\mathcal{E}(0)
$$

As $n \rightarrow \infty$, Eq. (47) suggests the exact solution for the model.

Theorem 5 The initial value problem given by Eq. (43) possesses at least one solution in the interval $[0, T]$.

Proof First, we define

$$
\left\{\begin{array}{l}
\mathcal{E}_{1}(t, \mathcal{T}(t))=\mathcal{T}(t)(1-\mathcal{T}(t))-\beta_{12} \mathcal{T}(t) \mathcal{H}(t)-\beta_{13} \mathcal{T}(t) \mathcal{E}(t) \\
\mathcal{E}_{2}(t, \mathcal{H}(t))=k_{2} \mathcal{H}(t)(1-\mathcal{H}(t))-\beta_{21} \mathcal{T}(t) \mathcal{H}(t) \\
\mathcal{E}_{3}(t, \mathcal{E}(t))=\frac{k_{3} \mathcal{T}(t) \mathcal{E}(t)}{\mathcal{T}(t)+s_{3}}-\beta_{31} \mathcal{T}(t) \mathcal{E}(t)-c_{3} \mathcal{E}(t)
\end{array}\right.
$$

where $\mathcal{E}_{1}(t, \mathcal{T}(t)), \mathcal{E}_{2}(t, \mathcal{H}(t))$, and $\mathcal{E}_{3}(t, \mathcal{E}(t))$ are contractions respect to $\mathcal{T}(t), \mathcal{H}(t)$, and $\mathcal{E}(t)$, respectively.

Moreover, we set

$$
\begin{aligned}
& \mathcal{N}_{1}=\sup \left\|_{\Psi_{a, b_{1}}} \mathcal{E}_{1}(t, \mathcal{T}(t))\right\|, \quad \mathcal{N}_{2}=\sup \left\|_{\Psi_{a, b_{2}}} \mathcal{E}_{2}(t, \mathcal{H}(t))\right\|, \\
& \mathcal{N}_{3}=\sup \left\|_{\Psi_{a, b_{3}}} \mathcal{E}_{3}(t, \mathcal{E}(t))\right\|,
\end{aligned}
$$

where

$$
\left\{\begin{array}{l}
\Psi_{a, b_{1}}=[t-a, t+a] \times\left[\mathcal{T}-b_{1}, \mathcal{T}+b_{1}\right]=A_{1} \times B_{1}, \\
\Psi_{a, b_{2}}=[t-a, t+a] \times\left[\mathcal{T}-b_{2}, \mathcal{H}+b_{2}\right]=A_{1} \times B_{2}, \\
\Psi_{a, b_{3}}=[t-a, t+a] \times\left[\mathcal{T}-b_{3}, \mathcal{E}+b_{3}\right]=A_{1} \times B_{3} .
\end{array}\right.
$$

Considering the Picard operator, we have

$$
\Theta:\left(\Psi_{a, b_{1}}, \Psi_{a, b_{2}}, \Psi_{a, b_{3}}\right) \rightarrow\left(\Psi_{a, b_{1}}, \Psi_{a, b_{2}}, \Psi_{a, b_{3}}\right)
$$

defined as follows

$$
\Theta \Omega(t)=\Omega_{0}(t)+\frac{1-\boldsymbol{\alpha}}{\mathrm{B}(\boldsymbol{\alpha})} \Delta(t, \Omega(t))+\frac{\boldsymbol{\alpha}}{\mathrm{B}(\boldsymbol{\alpha}) \Gamma(\boldsymbol{\alpha})} \int_{0}^{t} \Delta(\eta, \Omega(\eta))(t-\eta)^{\boldsymbol{\alpha}-1} d \eta
$$

where $\Omega(t)=(\mathcal{T}(t), \mathcal{H}(t), \mathcal{E}(t)), \Omega_{0}(t)=(\mathcal{T}(0), \mathcal{H}(0), \mathcal{E}(0))$, and $\Delta(t, \Omega(t))=\mathcal{E}_{1}(t, \mathcal{T}(t))$, $\mathcal{E}_{2}(t, \mathcal{H}(t)), \mathcal{E}_{3}(t, \mathcal{E}(t))$. 
Now we assume that all the solutions are bounded within a period of time:

$$
\begin{aligned}
& \|\Omega(t)\|_{\infty} \leq \max \left\{B_{1}, B_{2}, B_{3}, B_{4}\right\}, \\
& \left\|\Omega(t)-\Omega_{0}(t)\right\|_{\infty} \\
& \leq\left\|\frac{1-\boldsymbol{\alpha}}{\mathrm{B}(\boldsymbol{\alpha})} \Delta(t, \Omega(t))+\frac{\boldsymbol{\alpha}}{\mathrm{B}(\boldsymbol{\alpha}) \Gamma(\boldsymbol{\alpha})} \int_{0}^{t} \Delta(\eta, \Omega(\eta))(t-\eta)^{\boldsymbol{\alpha}-1} d \eta\right\| \\
& \leq \frac{1-\boldsymbol{\alpha}}{\mathrm{B}(\boldsymbol{\alpha})}\|\Delta(t, \Omega(t))\|+\frac{\boldsymbol{\alpha}}{\mathrm{B}(\boldsymbol{\alpha}) \Gamma(\boldsymbol{\alpha})} \int_{0}^{t}\|\Delta(\eta, \Omega(\eta))\|(t-\eta)^{\boldsymbol{\alpha}-1} d \eta \\
& \leq \frac{1-\boldsymbol{\alpha}}{\mathrm{B}(\boldsymbol{\alpha}) \Gamma(\boldsymbol{\alpha})} Z=\max \left\{B_{1}, B_{2}, B_{3}, B_{4}\right\}+\frac{\boldsymbol{\alpha}}{\mathrm{B}(\boldsymbol{\alpha}) \Gamma(\boldsymbol{\alpha})} Z \omega^{\boldsymbol{\alpha}} \\
& \leq \omega Z<B=\max \left\{B_{1}, B_{2}, B_{3}, B_{4}\right\},
\end{aligned}
$$

provided that

$$
\omega<\frac{B}{Z}
$$

The fixed point theorem of a Banach space together with the metric suggests that

$$
\begin{aligned}
&\left\|\Theta \Omega_{1}-\Theta \Omega_{2}\right\|_{\infty}=\sup _{t \in A}\left|\Omega_{1}-\Omega_{2}\right|, \\
&\left\|\Theta \Omega_{1}-\Theta \Omega_{2}\right\| \\
&=\left\|\Delta\left(t, \Omega_{1}(t)\right)-\Delta\left(t, \Omega_{2}(t)\right)\right\| \frac{1-\boldsymbol{\alpha}}{\mathrm{B}(\boldsymbol{\alpha})} \\
& \quad+\frac{\boldsymbol{\alpha}}{\mathrm{B}(\boldsymbol{\alpha}) \Gamma(\boldsymbol{\alpha})} \int_{0}^{t}\left\{\Delta\left(t, \Omega_{1}(t)\right)-\Delta\left(t, \Omega_{2}(t)\right)\right\}(t-\eta)^{\boldsymbol{\alpha}-1} d \eta \| \\
& \leq \frac{1-\boldsymbol{\alpha}}{\mathrm{B}(\boldsymbol{\alpha})}\left\|\Delta\left(t, \Omega_{1}(t)\right)-\Delta\left(t, \Omega_{2}(t)\right)\right\| \\
&+\frac{\boldsymbol{\alpha}}{\mathrm{B}(\boldsymbol{\alpha}) \Gamma(\boldsymbol{\alpha})} \int_{0}^{t}\left\|\Delta\left(t, \Omega_{1}(t)\right)-\Delta\left(t, \Omega_{2}(t)\right)\right\|(t-\eta)^{\boldsymbol{\alpha}-1} d \eta \\
& \leq \frac{1-\boldsymbol{\alpha}}{\mathrm{B}(\boldsymbol{\alpha})} \eta\left\|\Omega_{1}(t)-\Omega_{2}(t)\right\| \\
&+\frac{\boldsymbol{\alpha} \eta}{\mathrm{B}(\boldsymbol{\alpha}) \Gamma(\boldsymbol{\alpha})} \int_{0}^{t}\left\|\Omega_{1}(t)-\Omega_{2}(t)\right\|(t-\eta)^{\boldsymbol{\alpha}-1} d \eta \\
& \leq\left\{\frac{1-\boldsymbol{\alpha}}{\mathrm{B}(\boldsymbol{\alpha})} \eta+\frac{\boldsymbol{\alpha} \eta}{\mathrm{B}(\boldsymbol{\alpha}) \Gamma(\boldsymbol{\alpha})}\left(\frac{a^{\boldsymbol{\alpha}}}{\boldsymbol{\alpha}}\right)\right\}\left\|\Omega_{1}(t)-\Omega_{2}(t)\right\| \\
& \leq \eta \omega\left\|\Omega_{1}(t)-\Omega_{2}(t)\right\| .
\end{aligned}
$$

Since $\Omega$ is a contraction along with $\eta<1$, we must have $\omega \eta<1$. Therefore we conclude that $\Theta$ is a contraction operator. Finally, it is proved that (43) always possesses a unique solution. 


\subsection{Numerical method}

Consider the following fractional initial value problem:

$$
\begin{aligned}
& { }_{0}^{\mathrm{ABC}} \mathcal{D}_{t}^{\alpha} \phi(t)=\Xi(t, \phi(t)), \\
& \phi(0)=\phi_{0} .
\end{aligned}
$$

Employing the product integration rule, Ghanbari and Kumar [20] have developed an efficient scheme to obtain the approximate solution of (56), given by

$$
\phi_{n}=\phi_{0}+\frac{\alpha \hbar^{\alpha}}{\mathrm{B}(\alpha)}\left(p_{n} \Xi\left(t_{0}, \phi_{0}\right)+\sum_{\zeta=1}^{n} q_{-\zeta+n} \Xi\left(t_{\zeta}, \phi_{\zeta}\right)\right), \quad n \geq 1,
$$

where

$$
\begin{aligned}
& p_{n}=\frac{(n-1)^{\alpha+1}-n^{\alpha}(n-\alpha-1)}{\Gamma(\alpha+2)}, \\
& q_{j}= \begin{cases}\frac{1}{\Gamma(\alpha+2)}+\frac{1-\alpha}{\alpha \hbar^{\alpha}}, & j=0, \\
\frac{(j-1)^{\alpha+1}-2 j^{\alpha+1}+(j+1)^{\alpha+1}}{\Gamma(\alpha+2)}, & j=1,2, \ldots, n-1 .\end{cases}
\end{aligned}
$$

Using this numerical approximation, we get the following iterative scheme:

$$
\begin{aligned}
\mathcal{T}_{n}= & \mathcal{T}_{0}+\frac{\alpha \hbar^{\alpha}}{\mathrm{B}(\alpha)}\left(p_{n}\left[\mathcal{T}_{0}\left(1-\mathcal{T}_{0}\right)-\beta_{12} \mathcal{T}_{0} \mathcal{H}_{0}-\beta_{13} \mathcal{T}_{0} \mathcal{E}_{0}\right]\right. \\
& \left.+\sum_{\zeta=1}^{n} q_{n-i}\left[\mathcal{T}_{\zeta}\left(1-\mathcal{T}_{\zeta}\right)-\beta_{12} \mathcal{T}_{\zeta} \mathcal{H}_{\zeta}-\beta_{13} \mathcal{T}_{\zeta} \mathcal{E}_{\zeta}\right]\right) \\
\mathcal{H}_{n}= & \mathcal{H}_{0}+\frac{\alpha \hbar^{\alpha}}{\mathrm{B}(\alpha)}\left(p_{n}\left[k_{2} \mathcal{H}_{0}\left(1-\mathcal{H}_{0}\right)-\beta_{21} \mathcal{T}_{0} \mathcal{H}_{0}\right]\right. \\
& \left.+\sum_{\zeta=1}^{n} q_{n-i}\left[k_{2} \mathcal{H}_{\zeta}\left(1-\mathcal{H}_{\zeta}\right)-\beta_{21} \mathcal{T}_{\zeta} \mathcal{H}_{\zeta}\right]\right) \\
\mathcal{E}_{n}= & \mathcal{E}_{0}+\frac{\alpha \hbar^{\alpha}}{\mathrm{B}(\alpha)}\left(p_{n}\left[\frac{k_{3} \mathcal{T}_{0} \mathcal{E}_{0}}{\mathcal{T}_{0}+s_{3}}-\beta_{31} \mathcal{T}_{0} \mathcal{E}_{0}-c_{3} \mathcal{E}_{0}\right]\right. \\
& \left.+\sum_{\zeta=1}^{n} q_{n-i}\left[\frac{k_{3} \mathcal{T}_{\zeta} \mathcal{E}_{\zeta}}{\mathcal{T}_{\zeta}+s_{3}}-\beta_{31} \mathcal{T}_{\zeta} \mathcal{E}_{\zeta}-c_{3} \mathcal{E}_{\zeta}\right]\right) .
\end{aligned}
$$

\subsection{Numerical simulations}

Figures 7-9 are plotted to demonstrate the variation of state variables in model (43) when the scheme (59) is used for different values of $\alpha \in(0,1]$. In this simulations, we have considered the following values in the model: $\beta_{12}=1, \beta_{13}=2.5, k_{2}=0.6, \beta_{21}=1.5, k_{3}=4.5$, $s_{3}=1, a_{31}=0.2$, and $d_{3}=0.5$. In our performed numerical simulations, $t_{\text {final }}=500$ and $\hbar=0.001$. In Fig. 7, we take $\left.(\mathcal{T}(t), \mathcal{H}(t), \mathcal{E}(t))\right|_{t=0}=(0.1,0.1,0.1)$. In this case the model exhibits chaotic attractor behavior. Also, taking the initial conditions $\left.(\mathcal{T}(t), \mathcal{H}(t), \mathcal{E}(t))\right|_{t=0}=$ $(0.3,0.3,0.3)$ and $\beta_{12}=0.745$ the model shows the limit cycle behavior in Fig. 8. Moreover, 

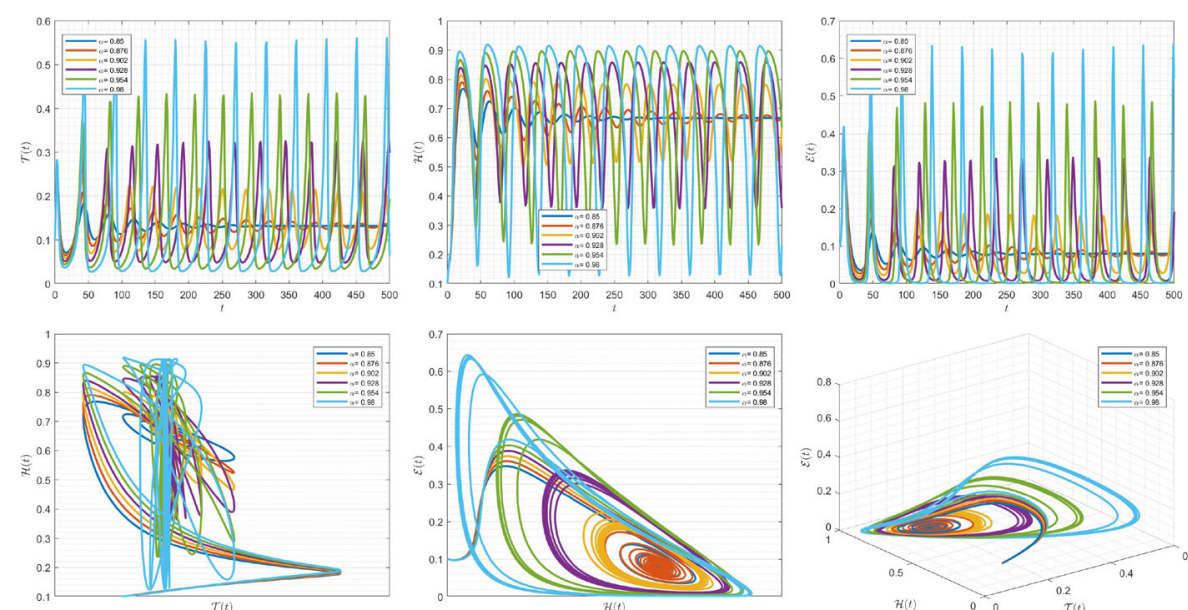

Figure 7 Simulations for solving (43) using (59) along with $(\mathcal{T}(0), \mathcal{H}(0), \mathcal{E}(0))=(0.1,0.1,0.1)$

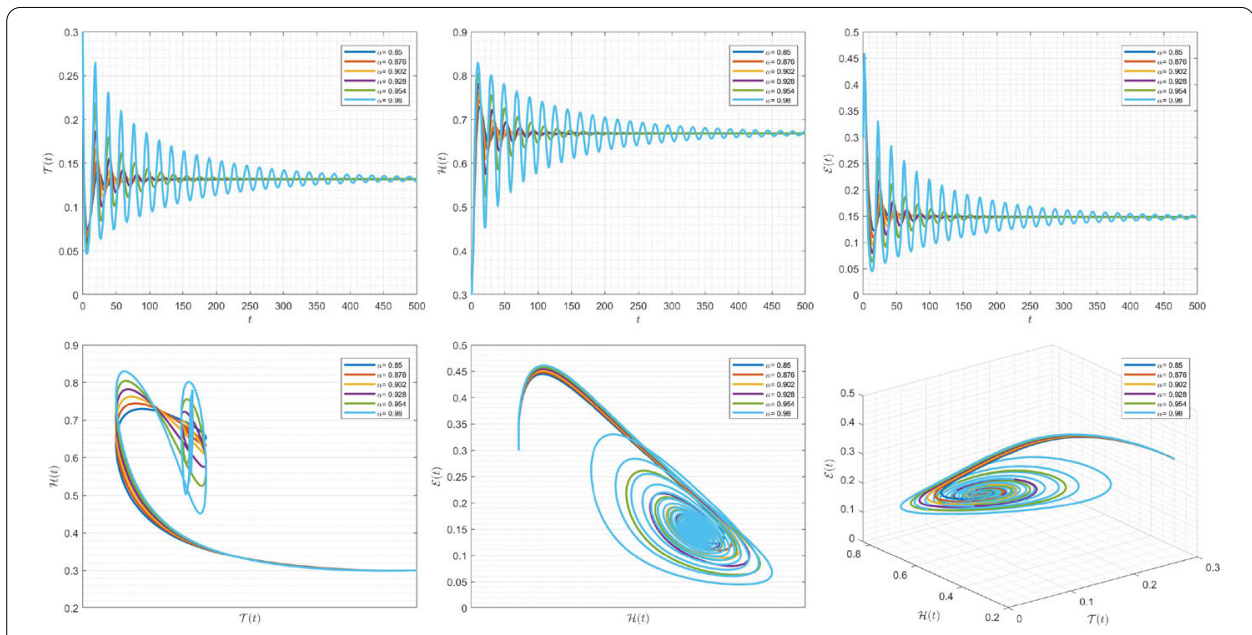

Figure 8 Simulations for solving (43) using (59) along with $(\mathcal{T}(0), \mathcal{H}(0), \mathcal{E}(0))=(0.3,0.3,0.3)$ and $\beta_{12}=0.745$
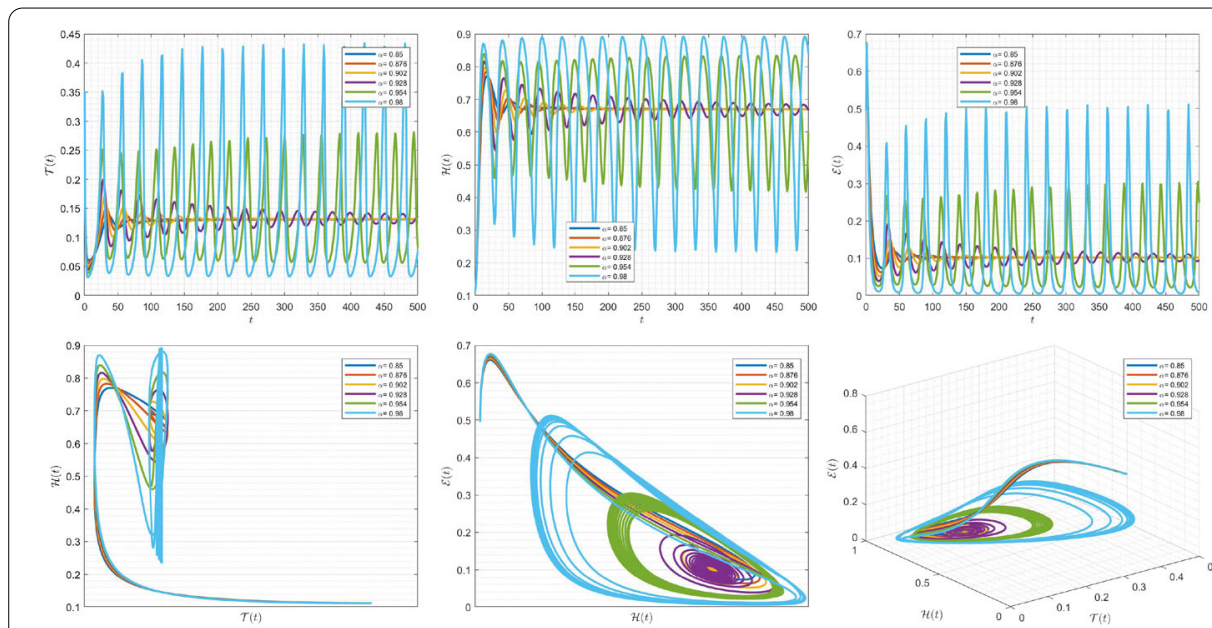

Figure 9 Simulations for solving (43) using (59) along with $(\mathcal{T}(0), \mathcal{H}(0), \mathcal{E}(0))=(0.3517,0.1115,0.4951)$ and $\beta_{12}=0.92$ 
for $\mathcal{T}(0)=0.3517, \mathcal{H}(0)=0.1115, \mathcal{E}(0)=0.4951$, and $\beta_{12}=0.920$, we get periodic orbit trajectories in the solutions as depicted in Fig. 9.

\section{The model via fractal-fractional derivative involving different laws}

In this section, we will use several well-known fractal-fractional derivatives in model (4).

\subsection{Power-law fractal-fractional derivative}

In this subsection, we replace the derivative in (4) by the fractal-fractional derivative with power-law:

$$
\begin{aligned}
& { }_{0}^{\mathrm{FF}-\mathrm{P}} \mathcal{D}_{t}^{\alpha, \tau} \mathcal{T}(t)=\mathcal{T}(t)(1-\mathcal{T}(t))-\beta_{12} \mathcal{T}(t) \mathcal{H}(t)-\beta_{13} \mathcal{T}(t) \mathcal{E}(t), \\
& { }_{0}^{\mathrm{FF}-\mathrm{P}} \mathcal{D}_{t}^{\alpha, \tau} \mathcal{H}(t)=k_{2} \mathcal{H}(t)(1-\mathcal{H}(t))-\beta_{21} \mathcal{T}(t) \mathcal{H}(t), \\
& { }_{0}^{\mathrm{FF}-\mathrm{P}} \mathcal{D}_{t}^{\alpha, \tau} \mathcal{E}(t)=\frac{k_{3} \mathcal{T}(t) \mathcal{E}(t)}{\mathcal{T}(t)+s_{3}}-\beta_{31} \mathcal{T}(t) \mathcal{E}(t)-c_{3} \mathcal{E}(t)
\end{aligned}
$$

where the fractal-fractional derivative with power-law of function $\phi(t)$ is defined as [11]

$$
{ }_{0}^{\mathrm{FF}-\mathrm{P}} \mathcal{I}_{t}^{\boldsymbol{\alpha}, \psi} \phi(t)=\frac{1}{\Gamma(n-\boldsymbol{\alpha})} \frac{d}{d t^{\psi}} \int_{0}^{t} \frac{\phi(\eta) d \eta}{(t-\eta)^{1-n+\alpha}}, \quad n-1<\boldsymbol{\alpha}, \psi \leq n \in \mathbb{N},
$$

and

$$
\frac{d}{d t^{\psi}} \phi(u)=\lim _{n \rightarrow \infty} \frac{\phi(t)-\phi(u)}{t^{\psi}-u^{\psi}} .
$$

By employing the corresponding inverse operator in Eq. (60) and then placing $t=t_{n+1}$ in the resultant we obtain the following recursive form:

$$
\begin{aligned}
\mathcal{T}_{n+1}(t)= & \mathcal{T}_{1}(0) \\
& +\frac{\tau}{\Gamma(\boldsymbol{\alpha})} \sum_{j=0}^{n} \int_{t_{j}}^{t_{j+1}} \eta^{\tau-1}\left(\mathcal{T}(\eta)(1-\mathcal{T}(\eta))-\beta_{12} \mathcal{T}(\eta) \mathcal{H}(\eta)-\beta_{13} \mathcal{T}(\eta) \mathcal{E}(\eta)\right) d \eta \\
\mathcal{H}_{n+1}(t)= & \mathcal{H}_{2}(0)+\frac{\tau}{\Gamma(\boldsymbol{\alpha})} \sum_{j=0}^{n} \int_{t_{j}}^{t_{j+1}} \eta^{\tau-1}\left(\eta_{1}\left[k_{2} \mathcal{H}(\eta)(1-\mathcal{H}(\eta))-\beta_{21} \mathcal{T}(\eta) \mathcal{H}(\eta)\right]\right) d \eta \\
\mathcal{E}_{n+1}(t)= & \mathcal{E}_{3}(0)+\frac{\tau}{\Gamma(\boldsymbol{\alpha})} \sum_{\zeta=0}^{n} \int_{t_{\zeta}}^{t_{\zeta+1}} \eta^{\tau-1}\left(\frac{k_{3} \mathcal{T}(\eta) \mathcal{E}(\eta)}{\mathcal{T}(\eta)+s_{3}}-\beta_{31} \mathcal{T}(\eta) \mathcal{E}(\eta)-c_{3} \mathcal{E}(\eta)\right) d \eta
\end{aligned}
$$

Now let us define the functions

$$
\begin{aligned}
& \mathcal{K}_{1}(\eta)=\eta^{\tau-1}\left(\mathcal{T}(\eta)(1-\mathcal{T}(\eta))-\beta_{12} \mathcal{T}(\eta) \mathcal{H}(\eta)-\beta_{13} \mathcal{T}(\eta) \mathcal{E}(\eta)\right) \\
& \mathcal{K}_{2}(\eta)=\eta^{\tau-1}\left(\eta_{1}\left[k_{2} \mathcal{H}(\eta)(1-\mathcal{H}(\eta))-\beta_{21} \mathcal{T}(\eta) \mathcal{H}(\eta)\right]\right) \\
& \mathcal{K}_{3}(\eta)=\eta^{\tau-1}\left(\frac{k_{3} \mathcal{T}(\eta) \mathcal{E}(\eta)}{\mathcal{T}(\eta)+s_{3}}-\beta_{31} \mathcal{T}(\eta) \mathcal{E}(\eta)-c_{3} \mathcal{E}(\eta)\right)
\end{aligned}
$$

These functions can be interpolated in $\left[t_{\zeta}, t_{\zeta+1}\right]$ as

$$
\mathcal{K}_{i}(\eta)=\frac{\eta-t_{\zeta-1}}{t_{\zeta}-t_{\zeta-1}} \mathcal{K}_{i}\left(t_{\zeta}\right)-\frac{\eta-t_{j}}{t_{\zeta}-t_{\zeta-1}} \mathcal{K}_{i}\left(t_{\zeta-1}\right)
$$


Thus we obtain

$$
\begin{aligned}
\mathcal{T}_{n+1}= & \mathcal{T}_{0}+\frac{\tau(\Delta t)^{\alpha}}{\Gamma(\boldsymbol{\alpha}+2)} \sum_{\zeta=0}^{n}\left[\mathcal { K } _ { 1 } ( t _ { \zeta } ) \left[(-\zeta+1+n)^{\alpha}(-\zeta+n+\boldsymbol{\alpha}+2)\right.\right. \\
& \left.-(-\zeta+n)^{\alpha}(-\zeta+n+2 \boldsymbol{\alpha}+2)\right] \\
& \left.-\mathcal{K}_{1}\left(t_{\zeta-1}\right)\left[(-\zeta+1+n)^{\alpha+1}-(-\zeta+n)^{\alpha}(-\zeta+n+\boldsymbol{\alpha}+1)\right]\right], \\
\mathcal{H}_{n+1}= & \mathcal{H}_{0}+\frac{\tau(\Delta t)^{\alpha}}{\Gamma(\boldsymbol{\alpha}+2)} \sum_{\zeta=0}^{n}\left[\mathcal { K } _ { 2 } ( t _ { \zeta } ) \left[(-\zeta+1+n)^{\alpha}(-\zeta+n+\boldsymbol{\alpha}+2)\right.\right. \\
& \left.-(-\zeta+n)^{\boldsymbol{\alpha}}(-\zeta+n+2 \boldsymbol{\alpha}+2)\right] \\
& \left.-\mathcal{K}_{2}\left(t_{\zeta-1}\right)\left[(-\zeta+1+n)^{\boldsymbol{\alpha}+1}-(-\zeta+n)^{\boldsymbol{\alpha}}(-\zeta+n+\boldsymbol{\alpha}+1)\right]\right], \\
\mathcal{E}_{n+1}= & \mathcal{E}_{0}+\frac{\tau(\Delta t)^{\alpha}}{\Gamma(\boldsymbol{\alpha}+2)} \sum_{\zeta=0}^{n}\left[\mathcal { K } _ { 3 } ( t _ { \zeta } ) \left[(-\zeta+1+n)^{\alpha}(-\zeta+n+\boldsymbol{\alpha}+2)\right.\right. \\
& \left.-(-\zeta+n)^{\alpha}(-\zeta+n+2 \boldsymbol{\alpha}+2)\right] \\
& \left.-\mathcal{K}_{3}\left(t_{\zeta-1}\right)\left[(-\zeta+1+n)^{\boldsymbol{\alpha}+1}-(-\zeta+n)^{\alpha}(-\zeta+n+\boldsymbol{\alpha}+1)\right]\right],
\end{aligned}
$$

where $\mathcal{K}_{j}, \zeta=1,2,3$, are given in (63).

\subsection{Numerical simulations}

Figures 10-12 demonstrate the variation of state variables in model (60) while applying scheme (65) for different values of $\tau \in(0,1]$, and $\alpha=0.95$. In this simulations, we considered the following values in the model: $\beta_{12}=1, \beta_{13}=2.5, k_{2}=0.6, \beta_{21}=1.5, k_{3}=4.5, s_{3}=1$, $a_{31}=0.2$, and $d_{3}=0.5$. In our numerical simulations, $t_{\text {final }}=500$ and $\hbar=0.001$. In Fig. 10, we put $\left.(\mathcal{T}(t), \mathcal{H}(t), \mathcal{E}(t))\right|_{t=0}=(0.1,0.1,0.1)$. In this case the model exhibits chaotic attractor behavior. Also, by imposing the initial conditions $\left.(\mathcal{T}(t), \mathcal{H}(t), \mathcal{E}(t))\right|_{t=0}=(0.3,0.3,0.3)$ and $\beta_{12}=0.745$ the model shows the limit cycle behavior in Fig. 11. Further, for $\mathcal{T}(0)=0.3517$,
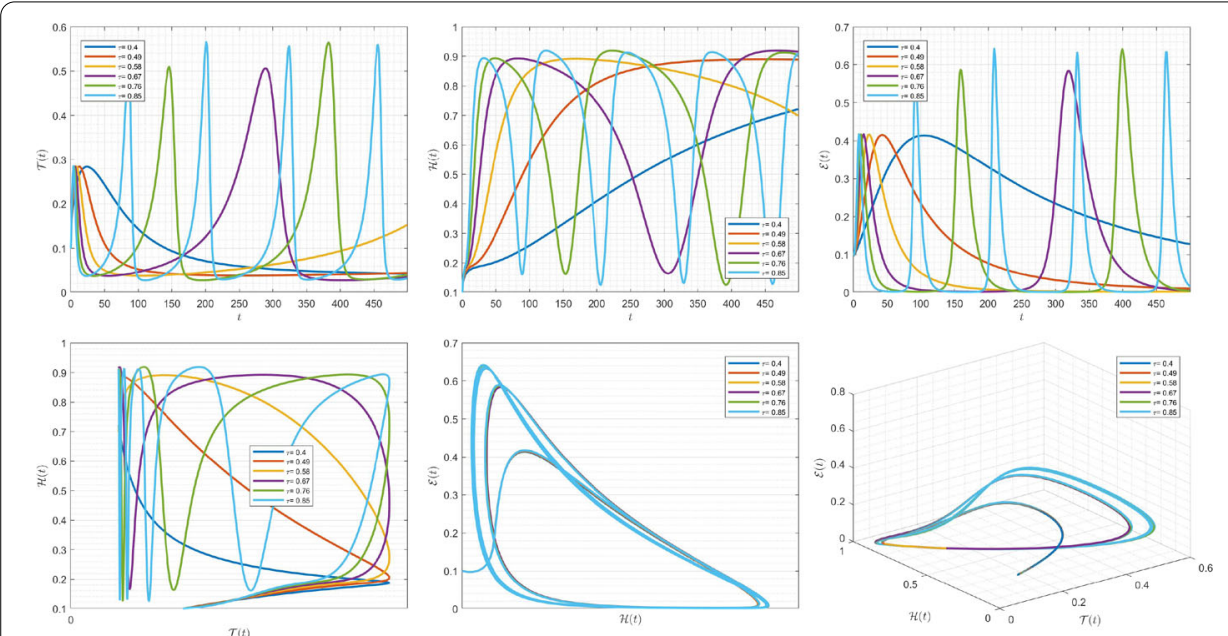

Figure 10 Simulations for solving (60) using (65) along with $(\mathcal{T}(0), \mathcal{H}(0), \mathcal{E}(0))=(0.1,0.1,0.1)$ 

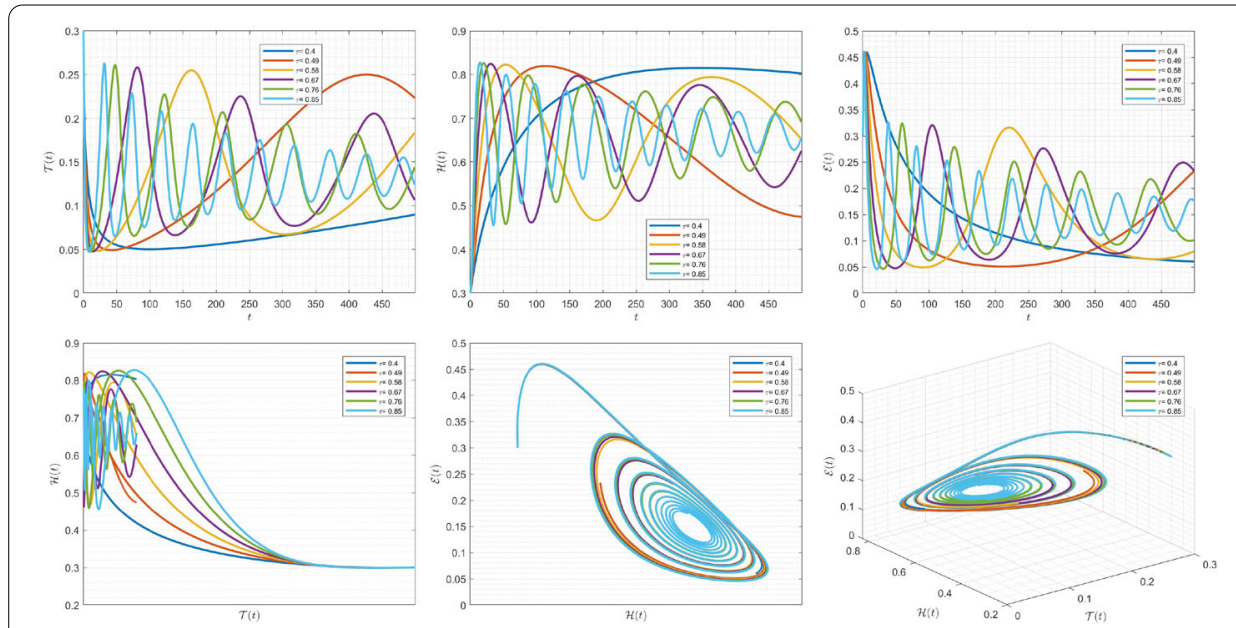

Figure 11 Simulations for solving (60) using (65) along with $(\mathcal{T}(0), \mathcal{H}(0), \mathcal{E}(0))=(0.3,0.3,0.3)$ and $\beta_{12}=0.745$

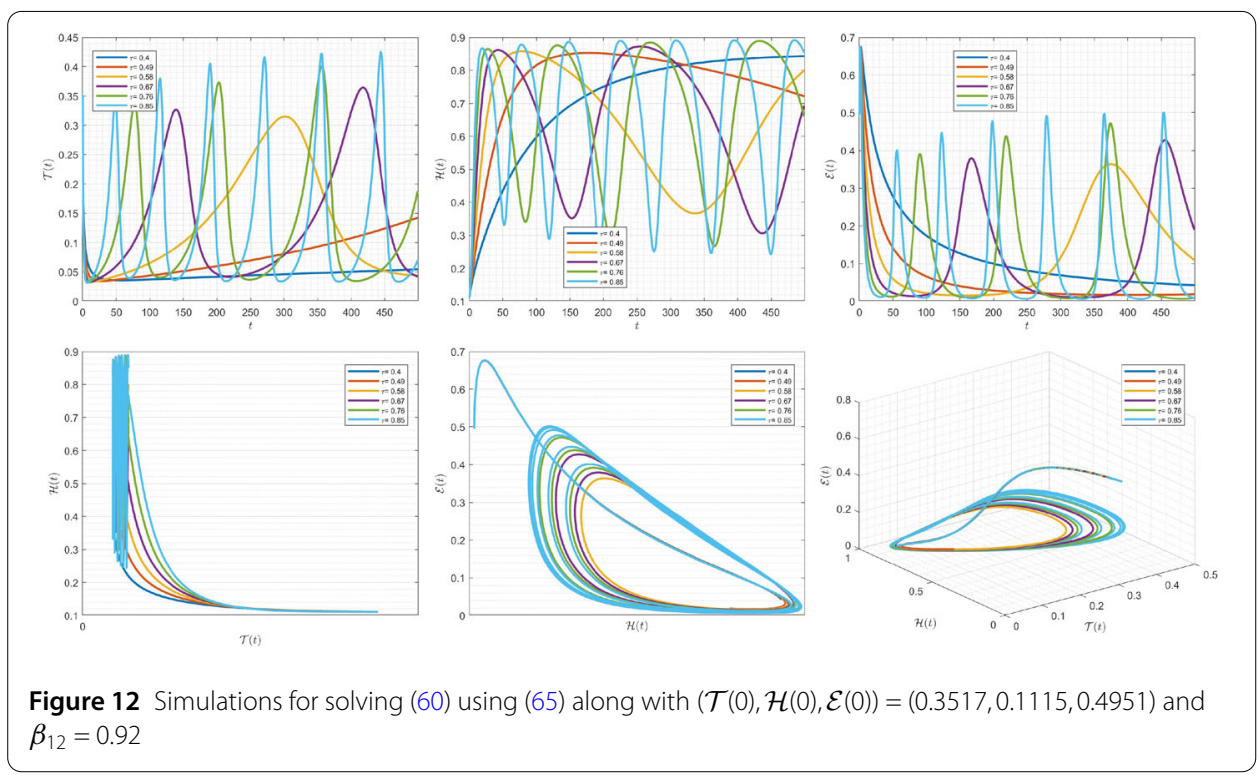

$\mathcal{H}(0)=0.1115, \mathcal{E}(0)=0.4951$, and $\beta_{12}=0.920$, Fig. 12 confirms the periodic orbit trajectories in the solutions.

\subsection{Exponential decay-law fractal-fractional derivative}

In this part, we replace the derivative in (4) by the fractal-fractional derivative with exponential decay-law:

$$
\begin{aligned}
& { }_{0}^{\mathrm{FF}-\mathrm{E}} \mathcal{D}_{t}^{\alpha, \tau} \mathcal{T}(t)=\mathcal{T}(t)(1-\mathcal{T}(t))-\beta_{12} \mathcal{T}(t) \mathcal{H}(t)-\beta_{13} \mathcal{T}(t) \mathcal{E}(t), \\
& { }_{0}^{\mathrm{FF}-\mathrm{E}} \mathcal{D}_{t}^{\alpha, \tau} \mathcal{H}(t)=k_{2} \mathcal{H}(t)(1-\mathcal{H}(t))-\beta_{21} \mathcal{T}(t) \mathcal{H}(t) \\
& { }_{0}^{\mathrm{FF}-\mathrm{E}} \mathcal{D}_{t}^{\alpha, \tau} \mathcal{E}(t)=\frac{k_{3} \mathcal{T}(t) \mathcal{E}(t)}{\mathcal{T}(t)+s_{3}}-\beta_{31} \mathcal{T}(t) \mathcal{E}(t)-c_{3} \mathcal{E}(t)
\end{aligned}
$$


where the fractal-fractional derivative with exponential decay law of a function $\phi(t)$ is defined as [11]

$$
{ }_{0}^{\mathrm{FF}-\mathrm{E}} \mathcal{I}_{t}^{\boldsymbol{\alpha}, \psi} \phi(t)=\frac{\mathrm{M}(\boldsymbol{\alpha})}{n-\boldsymbol{\alpha}} \frac{d}{d t^{\psi}} \int_{0}^{t} \phi(\eta) \exp \left[-\frac{\boldsymbol{\alpha}}{n-\boldsymbol{\alpha}}(t-\eta)\right] d \eta, \quad n-1<\boldsymbol{\alpha}, \psi \leq n \in \mathbb{N},
$$

and $\frac{d}{d t^{\psi}} \phi(u)$ is introduced in (62).

Applying the Caputo-Fabrizio integral to Eq. (69), we obtain

$$
\begin{aligned}
\mathcal{T}(t)= & \mathcal{T}(0)+\frac{\gamma_{1}}{\mathrm{M}(\boldsymbol{\alpha})} \mathcal{F}_{1}\left(t, \mathcal{T}(t), \mathcal{H}(t), \mathcal{E}(t), x_{4}(t)\right) \\
& +\frac{\gamma_{1} \boldsymbol{\alpha}}{\mathrm{M}(\boldsymbol{\alpha})} \int_{0}^{t} \mathcal{F}_{1}(\eta, \mathcal{T}(\eta), \mathcal{H}(\eta), \mathcal{E}(\eta)) d \eta \\
\mathcal{H}(t)= & \mathcal{H}(0)+\frac{\gamma_{2}}{\mathrm{M}(\boldsymbol{\alpha})} \mathcal{F}_{2}\left(t, \mathcal{T}(t), \mathcal{H}(t), \mathcal{E}(t), x_{4}(t)\right) \\
& +\frac{\gamma_{2} \boldsymbol{\alpha}}{\mathrm{M}(\boldsymbol{\alpha})} \int_{0}^{t} \mathcal{F}_{2}(\eta, \mathcal{T}(\eta), \mathcal{H}(\eta), \mathcal{E}(\eta)) d \eta \\
\mathcal{E}(t)= & \mathcal{E}(0)+\frac{\gamma_{3}}{\mathrm{M}(\boldsymbol{\alpha})} \mathcal{F}_{3}\left(t, \mathcal{T}(t), \mathcal{H}(t), \mathcal{E}(t), x_{4}(t)\right) \\
& +\frac{\gamma_{3} \boldsymbol{\alpha}}{\mathrm{M}(\boldsymbol{\alpha})} \int_{0}^{t} \mathcal{F}_{3}(\eta, \mathcal{T}(\eta), \mathcal{H}(\eta), \mathcal{E}(\eta)) d \eta
\end{aligned}
$$

Setting $t=t_{n+1}$ in (71), based on the proposed scheme in [11], we get

$$
\begin{aligned}
& \mathcal{T}_{n+1}=\mathcal{T}(0)+\frac{\tau}{\mathrm{M}(\boldsymbol{\alpha})} \mathcal{F}_{1}\left(t_{n}, \mathcal{T}_{n}, \mathcal{H}_{n}, \mathcal{E}_{n}\right)+\frac{\tau \boldsymbol{\alpha}}{\mathrm{M}(\boldsymbol{\alpha})} \int_{0}^{t_{n+1}} \mathcal{F}_{1}(\eta, \mathcal{T}(\eta), \mathcal{H}(\eta), \mathcal{E}(\eta)) d \eta \\
& \mathcal{H}_{n+1}=\mathcal{H}(0)+\frac{\tau}{\mathrm{M}(\boldsymbol{\alpha})} \mathcal{F}_{2}\left(t_{n}, \mathcal{T}_{n}, \mathcal{H}_{n}, \mathcal{E}_{n}\right)+\frac{\tau \boldsymbol{\alpha}}{\mathrm{M}(\boldsymbol{\alpha})} \int_{0}^{t_{n+1}} \mathcal{F}_{2}(\eta, \mathcal{T}(\eta), \mathcal{H}(\eta), \mathcal{E}(\eta)) d \eta \\
& \mathcal{E}_{n+1}=\mathcal{E}(0)+\frac{\tau}{\mathrm{M}(\boldsymbol{\alpha})} \mathcal{F}_{3}\left(t_{n}, \mathcal{T}_{n}, \mathcal{H}_{n}, \mathcal{E}_{n}\right)+\frac{\tau \boldsymbol{\alpha}}{\mathrm{M}(\boldsymbol{\alpha})} \int_{0}^{t_{n+1}} \mathcal{F}_{3}(\eta, \mathcal{T}(\eta), \mathcal{H}(\eta), \mathcal{E}(\eta)) d \eta
\end{aligned}
$$

Taking the difference between $\mathcal{T}_{n+1}$ and $\mathcal{T}_{n}$ yields

$$
\begin{aligned}
\mathcal{T}_{n+1}-\mathcal{T}_{n}= & \frac{\tau}{\mathrm{M}(\boldsymbol{\alpha})}\left[\mathcal{F}_{1}\left(t_{n}, \mathcal{T}_{n}, \mathcal{H}_{n}, \mathcal{E}_{n}\right)-\mathcal{F}_{1}\left(t_{n-1}, \mathcal{T}_{n-1}, \mathcal{H}_{n-1}, \mathcal{E}_{n-1}\right)\right] \\
& +\frac{\tau \boldsymbol{\alpha}}{\mathrm{M}(\boldsymbol{\alpha})} \int_{t_{n}}^{t_{n+1}} \mathcal{F}_{1}(\eta, \mathcal{T}(\eta), \mathcal{H}(\eta), \mathcal{E}(\eta)) d \eta \\
\mathcal{H}_{n+1}-\mathcal{H}_{n}= & \frac{\tau}{\mathrm{M}(\boldsymbol{\alpha})}\left[\mathcal{F}_{2}\left(t_{n}, \mathcal{T}_{n}, \mathcal{H}_{n}, \mathcal{E}_{n}\right)-\mathcal{F}_{2}\left(t_{n-1}, \mathcal{T}_{n-1}, \mathcal{H}_{n-1}, \mathcal{E}_{n-1}\right)\right] \\
& +\frac{\tau \boldsymbol{\alpha}}{\mathrm{M}(\boldsymbol{\alpha})} \int_{t_{n}}^{t_{n+1}} \mathcal{F}_{2}(\eta, \mathcal{T}(\eta), \mathcal{H}(\eta), \mathcal{E}(\eta)) d \eta \\
\mathcal{E}_{n+1}-\mathcal{E}_{n}= & \frac{\tau}{\mathrm{M}(\boldsymbol{\alpha})}\left[\mathcal{F}_{3}\left(t_{n}, \mathcal{T}_{n}, \mathcal{H}_{n}, \mathcal{E}_{n}\right)-\mathcal{F}_{3}\left(t_{n-1}, \mathcal{T}_{n-1}, \mathcal{H}_{n-1}, \mathcal{E}_{n-1}\right)\right] \\
& +\frac{\tau \boldsymbol{\alpha}}{\mathrm{M}(\boldsymbol{\alpha})} \int_{t_{n}}^{t_{n+1}} \mathcal{F}_{3}(\eta, \mathcal{T}(\eta), \mathcal{H}(\eta), \mathcal{E}(\eta)) d \eta
\end{aligned}
$$


Therefore the approximate solution to the problem can be determined using the following iterative procedures:

$$
\begin{aligned}
\mathcal{T}_{n+1}= & \mathcal{T}_{n}+\frac{\tau}{\mathrm{M}(\boldsymbol{\alpha})}\left[\mathcal{F}_{1}\left(t_{n}, \mathcal{T}_{n}, \mathcal{H}_{n}, \mathcal{E}_{n}\right)-\mathcal{F}_{1}\left(t_{n-1}, \mathcal{T}_{n-1}, \mathcal{H}_{n-1}, \mathcal{E}_{n-1}\right)\right] \\
& +\frac{\tau \boldsymbol{\alpha}}{\mathrm{M}(\boldsymbol{\alpha})}\left[\frac{3 \Delta}{2} \mathcal{F}_{1}\left(t_{n}, \mathcal{T}_{n}, \mathcal{H}_{n}, \mathcal{E}_{n}\right)-\frac{\Delta}{2} \mathcal{F}_{1}\left(t_{n-1}, x_{n-1}, \mathcal{H}_{n-1}, \mathcal{E}_{n-1}\right)\right], \\
\mathcal{H}_{n+1}= & \mathcal{H}_{n}+\frac{\tau}{\mathrm{M}(\boldsymbol{\alpha})}\left[\mathcal{F}_{2}\left(t_{n}, \mathcal{T}_{n}, \mathcal{H}_{n}, \mathcal{E}_{n}\right)-\mathcal{F}_{2}\left(t_{n-1}, \mathcal{T}_{n-1}, \mathcal{H}_{n-1}, \mathcal{E}_{n-1}\right)\right] \\
& +\frac{\tau \boldsymbol{\alpha}}{\mathrm{M}(\boldsymbol{\alpha})}\left[\frac{3 \Delta}{2} \mathcal{F}_{2}\left(t_{n}, \mathcal{T}_{n}, \mathcal{H}_{n}, \mathcal{E}_{n}\right)-\frac{\Delta}{2} \mathcal{F}_{2}\left(t_{n-1}, \mathcal{T}_{n-1}, \mathcal{Y}_{n-1}, \mathcal{E}_{n-1}\right)\right], \\
\mathcal{E}_{n+1}= & \mathcal{E}_{n}+\frac{\tau}{\mathrm{M}(\boldsymbol{\alpha})}\left[\mathcal{F}_{3}\left(t_{n}, \mathcal{T}_{n}, \mathcal{H}_{n}, \mathcal{E}_{n}\right)-\mathcal{F}_{3}\left(t_{n-1}, \mathcal{T}_{n-1}, \mathcal{H}_{n-1}, \mathcal{E}_{n-1}\right)\right] \\
& +\frac{\tau \boldsymbol{\alpha}}{\mathrm{M}(\boldsymbol{\alpha})}\left[\frac{3 \Delta}{2} \mathcal{F}_{3}\left(t_{n}, \mathcal{T}_{n}, \mathcal{H}_{n}, \mathcal{E}_{n}\right)-\frac{\Delta}{2} \mathcal{F}_{3}\left(t_{n-1}, \mathcal{T}_{n-1}, \mathcal{H}_{n-1}, \mathcal{E}_{n-1}\right)\right],
\end{aligned}
$$

where $\mathcal{F}_{j}, \zeta=1,2,3$, are given in (63).

\subsection{Numerical simulations}

Figures 13-15 demonstrate the variation of state variables in model (69) when scheme (74) is applied for different values of $\tau \in(0,1]$ and $\alpha=0.95$. In this simulations, we considered the following values in the model: $\beta_{12}=1, \beta_{13}=2.5, k_{2}=0.6, \beta_{21}=1.5, k_{3}=4.5$, $s_{3}=1, a_{31}=0.2$, and $d_{3}=0.5$. In our numerical simulations, $t_{\text {final }}=500$ and $\hbar=0.001$. In Fig. 13, we take $\left.(\mathcal{T}(t), \mathcal{H}(t), \mathcal{E}(t))\right|_{t=0}=(0.1,0.1,0.1)$. In this case the model exhibits chaotic attractor behavior. Further, for $\left.(\mathcal{T}(t), \mathcal{H}(t), \mathcal{E}(t))\right|_{t=0}=(0.3,0.3,0.3)$ and $\beta_{12}=0.745$, the model shows the limit cycle behavior in Fig. 14. Also, letting $\mathcal{T}(0)=0.3517, \mathcal{H}(0)=0.1115$, $\mathcal{E}(0)=0.4951$, and $\beta_{12}=0.920$, Fig. 15 confirms the periodic orbit trajectories in the solutions.
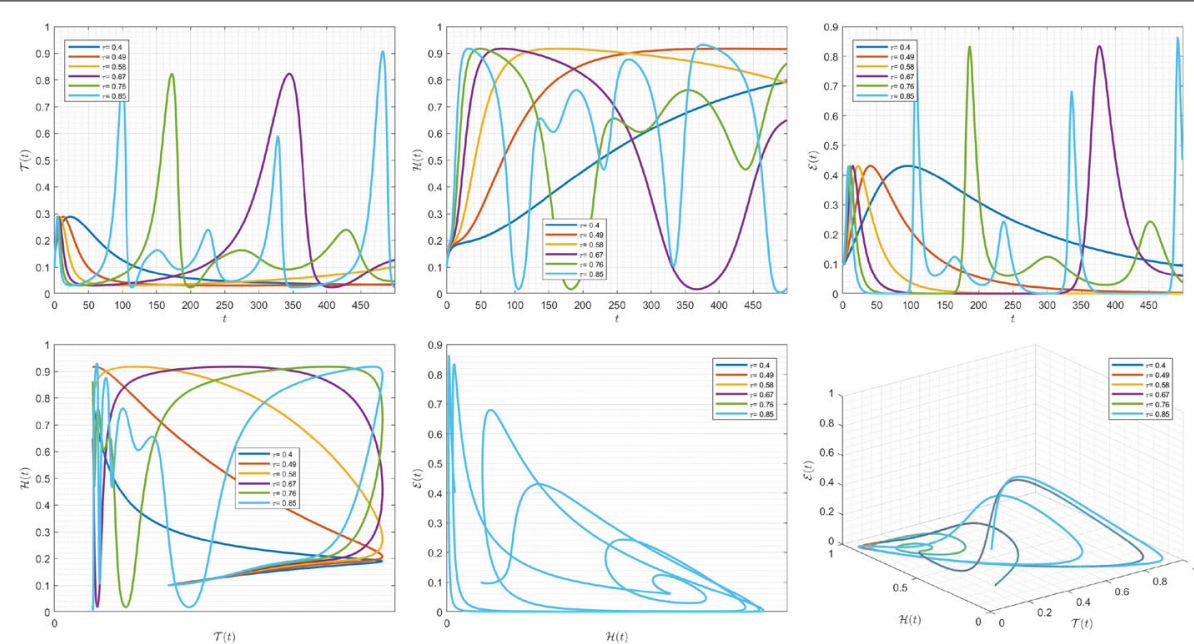

Figure 13 Simulations for solving (69) using (74) along with $(\mathcal{T}(0), \mathcal{H}(0), \mathcal{E}(0))=(0.1,0.1,0.1)$ 

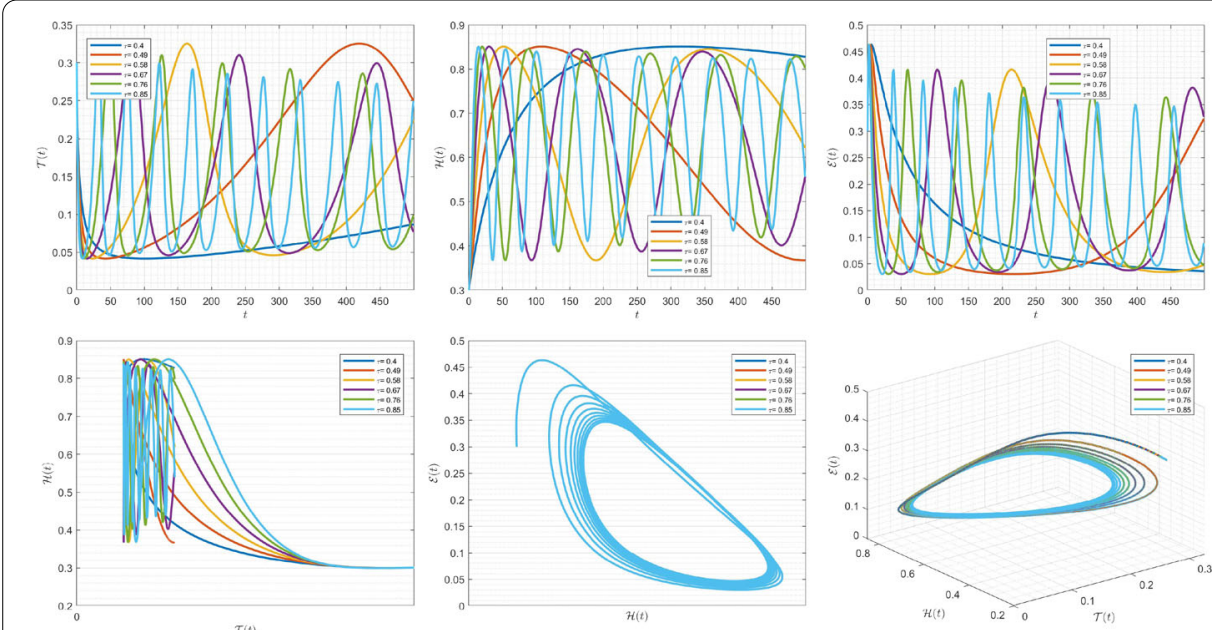

Figure 14 Simulations for solving (69) using (74) along with $(\mathcal{T}(0), \mathcal{H}(0), \mathcal{E}(0))=(0.3,0.3,0.3)$ and $\beta_{12}=0.745$
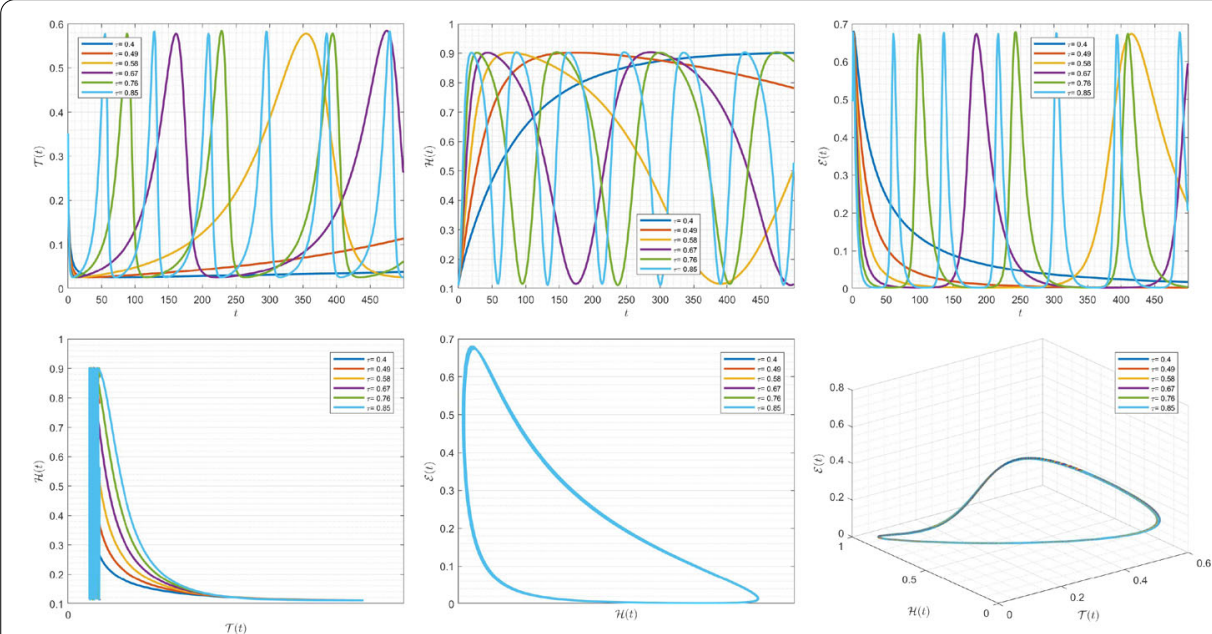

Figure 15 Simulations for solving (69) using (74) along with $(\mathcal{T}(0), \mathcal{H}(0), \mathcal{E}(0))=(0.3517,0.1115,0.4951)$ and $\beta_{12}=0.92$

\subsection{Mittag-Leffler-law fractal-fractional derivative}

In this subsection, we use the fractal-fractional derivative with Mittag-Leffler law in (4).

So, we achieve

$$
\begin{aligned}
& { }_{0}^{\mathrm{FF}-\mathrm{ABC}} \mathcal{D}_{t}^{\alpha, \tau} \mathcal{T}(t)=\mathcal{T}(t)(1-\mathcal{T}(t))-\beta_{12} \mathcal{T}(t) \mathcal{H}(t)-\beta_{13} \mathcal{T}(t) \mathcal{E}(t), \\
& { }_{0}^{\mathrm{FF}-\mathrm{ABC}} \mathcal{D}_{t}^{\alpha, \tau} \mathcal{H}(t)=k_{2} \mathcal{H}(t)(1-\mathcal{H}(t))-\beta_{21} \mathcal{T}(t) \mathcal{H}(t), \\
& { }_{0}^{\mathrm{FF}-\mathrm{ABC}} \mathcal{D}_{t}^{\alpha, \tau} \mathcal{E}(t)=\frac{k_{3} \mathcal{T}(t) \mathcal{E}(t)}{\mathcal{T}(t)+s_{3}}-\beta_{31} \mathcal{T}(t) \mathcal{E}(t)-c_{3} \mathcal{E}(t),
\end{aligned}
$$


where the fractal-fractional derivative with Mittag-Leffler law of a unction $\phi(t)$ is defined as [11]

$$
\begin{aligned}
& 0^{\mathrm{FF}-\mathrm{ABC}} \mathcal{D}_{t}^{\boldsymbol{\alpha}, \tau} \phi(t) \\
& \quad=\frac{\mathrm{B}(\boldsymbol{\alpha})}{n-\boldsymbol{\alpha}} \frac{d}{d t^{\psi}} \int_{0}^{t} \phi(\eta) E_{\boldsymbol{\alpha}}\left[-\frac{\boldsymbol{\alpha}}{n-\boldsymbol{\alpha}}(t-\eta)^{\alpha}\right] d \eta, \quad n-1<\boldsymbol{\alpha}, \psi \leq n \in \mathbb{N},
\end{aligned}
$$

and $\frac{d}{d t^{\psi}} \phi(u)$ is introduced in (62).

Applying the Atangana-Baleanu integral to (75), we obtain

$$
\begin{aligned}
\mathcal{T}(t)= & \mathcal{T}(0)+\frac{\tau t^{\tau-1}(1-\boldsymbol{\alpha})}{\Gamma(\boldsymbol{\alpha})} \mathcal{F}_{1}\left(t, \mathcal{T}(t), \mathcal{H}(t), \mathcal{E}(t), x_{4}(t)\right) \\
& +\frac{\tau \boldsymbol{\alpha}}{\Gamma(\boldsymbol{\alpha}) \mathrm{B}(\boldsymbol{\alpha})} \int_{0}^{t} \delta^{\tau-1} \mathcal{F}_{1}(\eta, \mathcal{T}(\eta), \mathcal{H}(\eta), \mathcal{E}(\eta))(t-\eta)^{\boldsymbol{\alpha}-1} d \eta \\
\mathcal{H}(t)= & \mathcal{H}(0)+\frac{\tau t^{\tau-1}(1-\boldsymbol{\alpha})}{\Gamma(\boldsymbol{\alpha})} \mathcal{F}_{2}\left(t, \mathcal{T}(t), \mathcal{H}(t), \mathcal{E}(t), x_{4}(t)\right) \\
& +\frac{\tau \boldsymbol{\alpha}}{\Gamma(\boldsymbol{\alpha}) \mathrm{B}(\boldsymbol{\alpha})} \int_{0}^{t} \delta^{\tau-1} \mathcal{F}_{2}(\eta, \mathcal{T}(\eta), \mathcal{H}(\eta), \mathcal{E}(\eta))(t-\eta)^{\boldsymbol{\alpha}-1} d \eta, \\
\mathcal{E}(t)= & \mathcal{E}(0)+\frac{\tau t^{\tau-1}(1-\boldsymbol{\alpha})}{\Gamma(\boldsymbol{\alpha})} \mathcal{F}_{3}\left(t, \mathcal{T}(t), \mathcal{H}(t), \mathcal{E}(t), x_{4}(t)\right) \\
& +\frac{\tau \boldsymbol{\alpha}}{\Gamma(\boldsymbol{\alpha}) \mathrm{B}(\boldsymbol{\alpha})} \int_{0}^{t} \delta^{\tau-1} \mathcal{F}_{3}(\eta, \mathcal{T}(\eta), \mathcal{H}(\eta), \mathcal{E}(\eta))(t-\eta)^{\boldsymbol{\alpha}-1} d \eta .
\end{aligned}
$$

Based on the scheme proposed in [11], we get

$$
\begin{aligned}
\mathcal{T}_{n+1}= & \mathcal{T}_{0}+\frac{\tau t_{n}^{\alpha-1}(1-\boldsymbol{\alpha})}{\Gamma(\boldsymbol{\alpha})} \mathcal{F}_{1}\left(t_{n}, \mathcal{T}_{n}, \mathcal{H}_{n}, \mathcal{E}_{n}\right) \\
& +\frac{\tau \boldsymbol{\alpha}}{\Gamma(\boldsymbol{\alpha}) \mathrm{B}(\boldsymbol{\alpha})} \sum_{\zeta=0}^{n} \int_{t_{\zeta}}^{t_{j+1}} \delta^{\tau-1} \mathcal{F}_{1}(\eta, \mathcal{T}(\eta), \mathcal{H}(\eta), \mathcal{E}(\eta))\left(t_{n+1}-\eta\right)^{\boldsymbol{\alpha}-1} d \eta, \\
\mathcal{H}_{n+1}= & \mathcal{H}_{0}+\frac{\tau t_{n}^{\alpha-1}(1-\boldsymbol{\alpha})}{\Gamma(\boldsymbol{\alpha})} \mathcal{F}_{2}\left(t_{n}, \mathcal{T}_{n}, \mathcal{H}_{n}, \mathcal{E}_{n}\right) \\
& +\frac{\tau \boldsymbol{\alpha}}{\Gamma(\boldsymbol{\alpha}) \mathrm{B}(\boldsymbol{\alpha})} \sum_{\zeta=0}^{n} \int_{t_{\zeta}}^{t_{j+1}} \delta^{\tau-1} \mathcal{F}_{2}(\eta, \mathcal{T}(\eta), \mathcal{H}(\eta), \mathcal{E}(\eta))\left(t_{n+1}-\eta\right)^{\boldsymbol{\alpha}-1} d \eta, \\
\mathcal{E}_{n+1}= & \mathcal{E}_{0}+\frac{\tau t_{n}^{\alpha-1}(1-\boldsymbol{\alpha})}{\Gamma(\boldsymbol{\alpha})} \mathcal{F}_{3}\left(t_{n}, \mathcal{T}_{n}, \mathcal{H}_{n}, \mathcal{E}_{n}\right) \\
& +\frac{\tau \boldsymbol{\alpha}}{\Gamma(\boldsymbol{\alpha}) \mathrm{B}(\boldsymbol{\alpha})} \sum_{\zeta=0}^{n} \int_{t_{\zeta}}^{t_{j+1}} \delta^{\tau-1} \mathcal{F}_{3}(\eta, \mathcal{T}(\eta), \mathcal{H}(\eta), \mathcal{E}(\eta))\left(t_{n+1}-\eta\right)^{\boldsymbol{\alpha}-1} d \eta .
\end{aligned}
$$

Now using the Lagrange polynomial piecewise interpolation given by Eq. (64), we obtain

$$
\begin{aligned}
\mathcal{T}_{n+1}= & \mathcal{T}_{0}+\frac{\tau t_{n}^{\alpha-1}(1-\boldsymbol{\alpha})}{\Gamma(\boldsymbol{\alpha})} \mathcal{F}_{1}\left(t_{n}, \mathcal{T}_{n}, \mathcal{H}_{n}, \mathcal{E}_{n}\right) \\
& +\frac{\tau \hbar^{\alpha}}{\Gamma(\boldsymbol{\alpha}+2) \mathrm{B}(\boldsymbol{\alpha})} \sum_{\zeta=0}^{n}\left[t _ { \zeta } ^ { \alpha - 1 } \mathcal { F } _ { 1 } ( t _ { \zeta } , \mathcal { T } _ { \zeta } , \mathcal { H } _ { \zeta } , \mathcal { E } _ { \zeta } ) \left[(-\zeta+1+n)^{\alpha}(-\zeta+n+\boldsymbol{\alpha}+2)\right.\right.
\end{aligned}
$$




$$
\begin{aligned}
& \left.-(-\zeta+n)^{\alpha}(-\zeta+n+2 \boldsymbol{\alpha}+2)\right] \\
& \left.-t_{\zeta-1}^{\alpha-1} \mathcal{F}_{1}\left(t_{\zeta-1}, \mathcal{T}_{\zeta-1}, \mathcal{H}_{\zeta-1}, \mathcal{E}_{\zeta-1}\right)\left[(-\zeta+1+n)^{\alpha+1}-(-\zeta+n)^{\alpha}(-\zeta+n+\boldsymbol{\alpha}+1)\right]\right] \\
\mathcal{H}_{n+1}= & \mathcal{H}_{0}+\frac{\tau t_{n}^{\alpha-1}(1-\boldsymbol{\alpha})}{\Gamma(\boldsymbol{\alpha})} \mathcal{F}_{2}\left(t_{n}, \mathcal{T}_{n}, \mathcal{H}_{n}, \mathcal{E}_{n}\right) \\
& +\frac{\tau \hbar^{\alpha}}{\Gamma(\boldsymbol{\alpha}+2) \mathrm{B}(\boldsymbol{\alpha})} \sum_{\zeta=0}^{n}\left[t _ { \zeta } ^ { \alpha - 1 } \mathcal { F } _ { 2 } ( t _ { \zeta } , \mathcal { T } _ { \zeta } , \mathcal { H } _ { \zeta } , \mathcal { E } _ { \zeta } ) \left[(-\zeta+1+n)^{\alpha}(-\zeta+n+\boldsymbol{\alpha}+2)\right.\right. \\
& \left.-(-\zeta+n)^{\alpha}(-\zeta+n+2 \boldsymbol{\alpha}+2)\right] \\
& \left.-t_{\zeta-1}^{\alpha-1} \mathcal{F}_{2}\left(t_{\zeta-1}, \mathcal{T}_{\zeta-1}, \mathcal{H}_{\zeta-1}, \mathcal{E}_{\zeta-1}\right)\left[(-\zeta+1+n)^{\alpha+1}-(-\zeta+n)^{\alpha}(-\zeta+n+\boldsymbol{\alpha}+1)\right]\right] \\
\mathcal{E}_{n+1}= & \mathcal{E}_{0}+\frac{\tau t_{n}^{\alpha-1}(1-\boldsymbol{\alpha})}{\Gamma(\boldsymbol{\alpha})} \mathcal{F}_{3}\left(t_{n}, \mathcal{T}_{n}, \mathcal{H}_{n}, \mathcal{E}_{n}\right) \\
& +\frac{\tau \hbar^{\alpha}}{\Gamma(\boldsymbol{\alpha}+2) \mathrm{B}(\boldsymbol{\alpha})} \sum_{\zeta=0}^{n}\left[t _ { \zeta } ^ { \alpha - 1 } \mathcal { F } _ { 3 } ( t _ { \zeta } , \mathcal { T } _ { \zeta } , \mathcal { H } _ { \zeta } , \mathcal { E } _ { \zeta } ) \left[(-\zeta+1+n)^{\alpha}(-\zeta+n+\boldsymbol{\alpha}+2)\right.\right. \\
& \left.-(-\zeta+n)^{\alpha}(-\zeta+n+2 \boldsymbol{\alpha}+2)\right] \\
& \left.-t_{\zeta-1}^{\alpha-1} \mathcal{F}_{3}\left(t_{\zeta-1}, \mathcal{T}_{\zeta-1}, \mathcal{H}_{\zeta-1}, \mathcal{E}_{\zeta-1}\right)\left[(-\zeta+1+n)^{\alpha+1}-(-\zeta+n)^{\alpha}(-\zeta+n+\boldsymbol{\alpha}+1)\right]\right],
\end{aligned}
$$

where

$$
\begin{aligned}
& \mathcal{F}_{1}(\eta, \mathcal{T}(\eta), \mathcal{H}(\eta), \mathcal{E}(\eta))=\mathcal{T}(\eta)(1-\mathcal{T}(\eta))-\beta_{12} \mathcal{T}(\eta) \mathcal{H}(\eta)-\beta_{13} \mathcal{T}(\eta) \mathcal{E}(\eta), \\
& \mathcal{F}_{2}(\eta, \mathcal{T}(\eta), \mathcal{H}(\eta), \mathcal{E}(\eta))=\eta_{1}\left[k_{2} \mathcal{H}(\eta)(1-\mathcal{H}(\eta))-\beta_{21} \mathcal{T}(\eta) \mathcal{H}(\eta)\right], \\
& \mathcal{F}_{3}(\eta, \mathcal{T}(\eta), \mathcal{H}(\eta), \mathcal{E}(\eta))=\frac{k_{3} \mathcal{T}(\eta) \mathcal{E}(\eta)}{\mathcal{T}(\eta)+s_{3}}-\beta_{31} \mathcal{T}(\eta) \mathcal{E}(\eta)-c_{3} \mathcal{E}(\eta)
\end{aligned}
$$

\subsection{Numerical simulations}

Figures 16-18 demonstrate the variation of state variables in model (75) when scheme (79) is utilized for different values of $\tau \in(0,1]$ and $\alpha=0.95$. In this simulations, we considered the following values in the model: $\beta_{12}=1, \beta_{13}=2.5, k_{2}=0.6, \beta_{21}=1.5, k_{3}=4.5, s_{3}=1, a_{31}=$
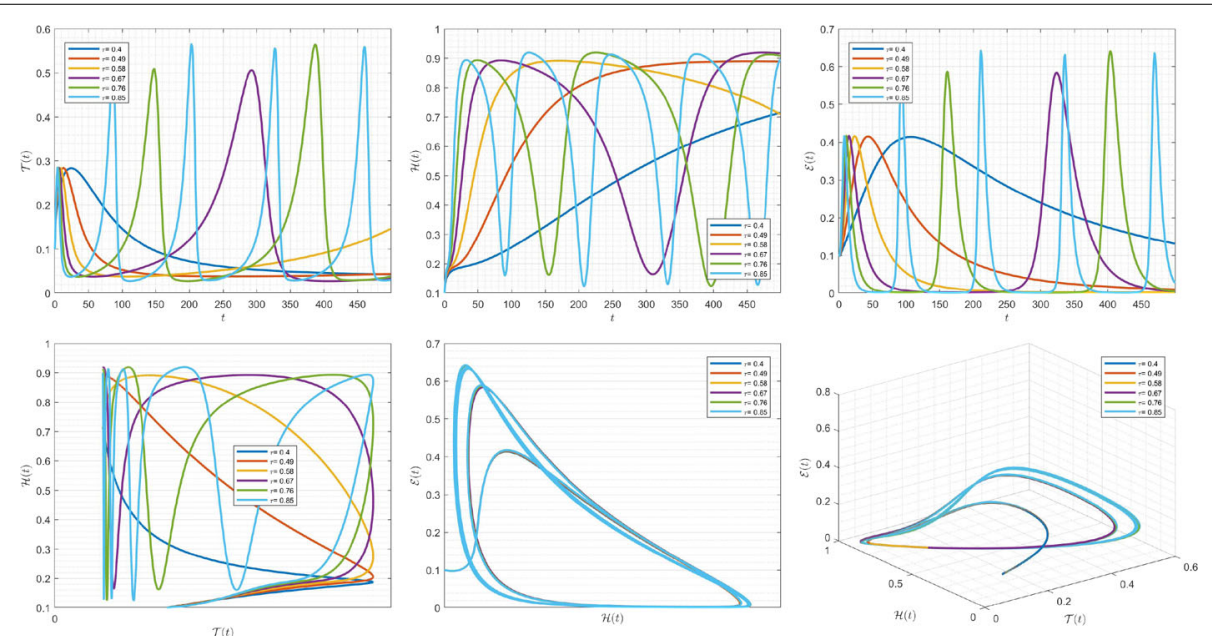

Figure 16 Simulations for solving (75) using (79) along with $(\mathcal{T}(0), \mathcal{H}(0), \mathcal{E}(0))=(0.1,0.1,0.1)$ 

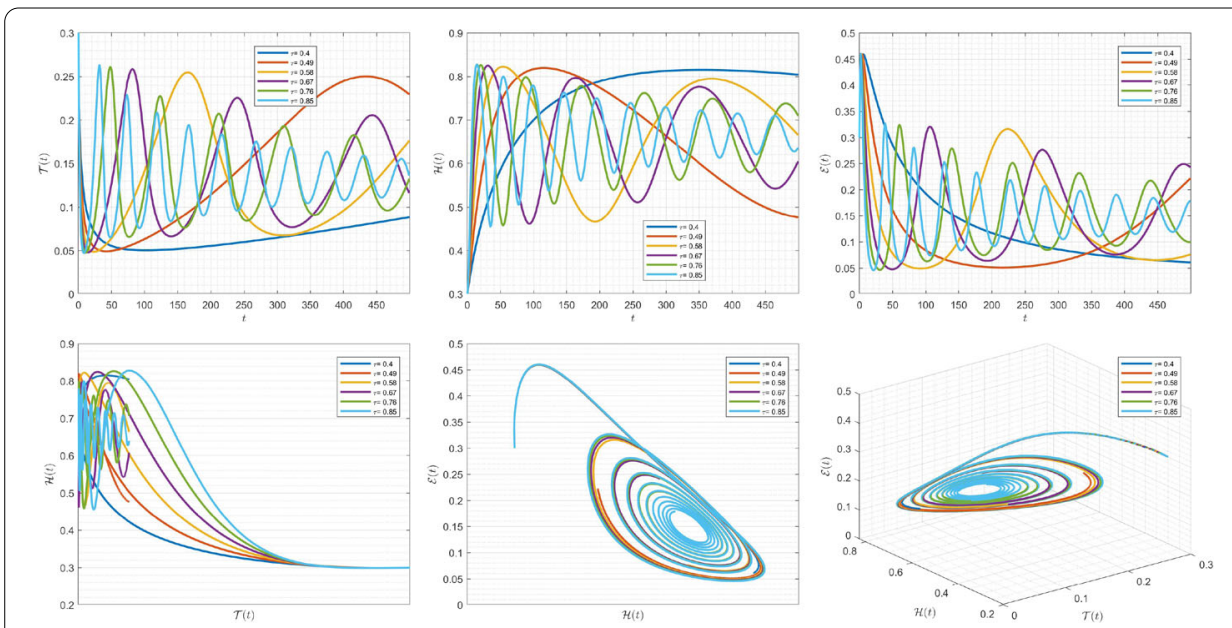

Figure 17 Simulations for solving (75) using (79) along with $(\mathcal{T}(0), \mathcal{H}(0), \mathcal{E}(0))=(0.3,0.3,0.3)$ and $\beta_{12}=0.745$
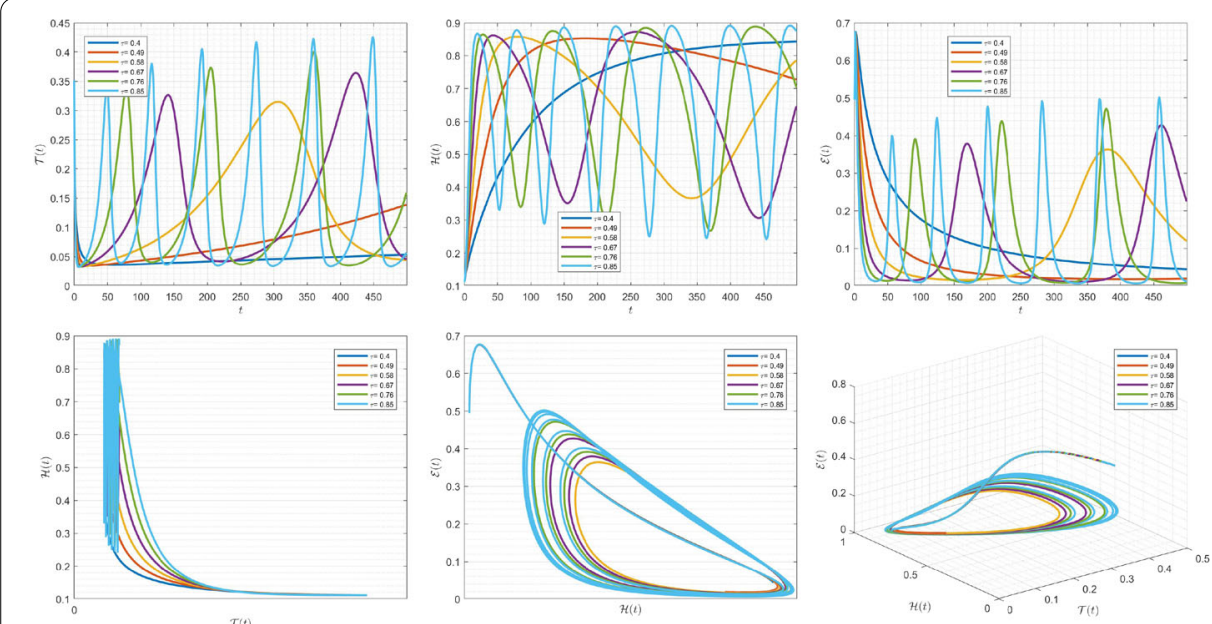

Figure 18 Simulations for solving (75) using (79) along with $(\mathcal{T}(0), \mathcal{H}(0), \mathcal{E}(0))=(0.3517,0.1115,0.4951)$ and $\beta_{12}=0.92$

0.2 , and $d_{3}=0.5$. In our numerical simulations, $t_{\text {final }}=500$ and $\hbar=0.001$. In Fig. 16, we take $\left.(\mathcal{T}(t), \mathcal{H}(t), \mathcal{E}(t))\right|_{t=0}=(0.1,0.1,0.1)$. In this case the model exhibits chaotic attractor behavior. Also, by taking into consideration $\left.(\mathcal{T}(t), \mathcal{H}(t), \mathcal{E}(t))\right|_{t=0}=(0.3,0.3,0.3)$ and $\beta_{12}=$ 0.745 the model shows the limit cycle behavior in Fig. 17, whereast for $\mathcal{T}(0)=0.3517$, $\mathcal{H}(0)=0.1115, \mathcal{E}(0)=0.4951$, and $\beta_{12}=0.920$, we get periodic orbit trajectories in the solutions as depicted in Fig. 18.

In Figs. 19 and 20, we also investigate the sensitivity of the state variables when two parameters $\beta_{12}$ and $c_{3}$ change, respectively. In these two figures, we can see that changes in each of these parameters cause changes that are somewhat meaningful and constructive in the behavior of variables. As a biological conclusion, we can point to the fact that the stability in the model can only be achieved when the recruitment rate of effector cells is greater than the rate of inactivation by cancer cells. In other words, if the immune system 

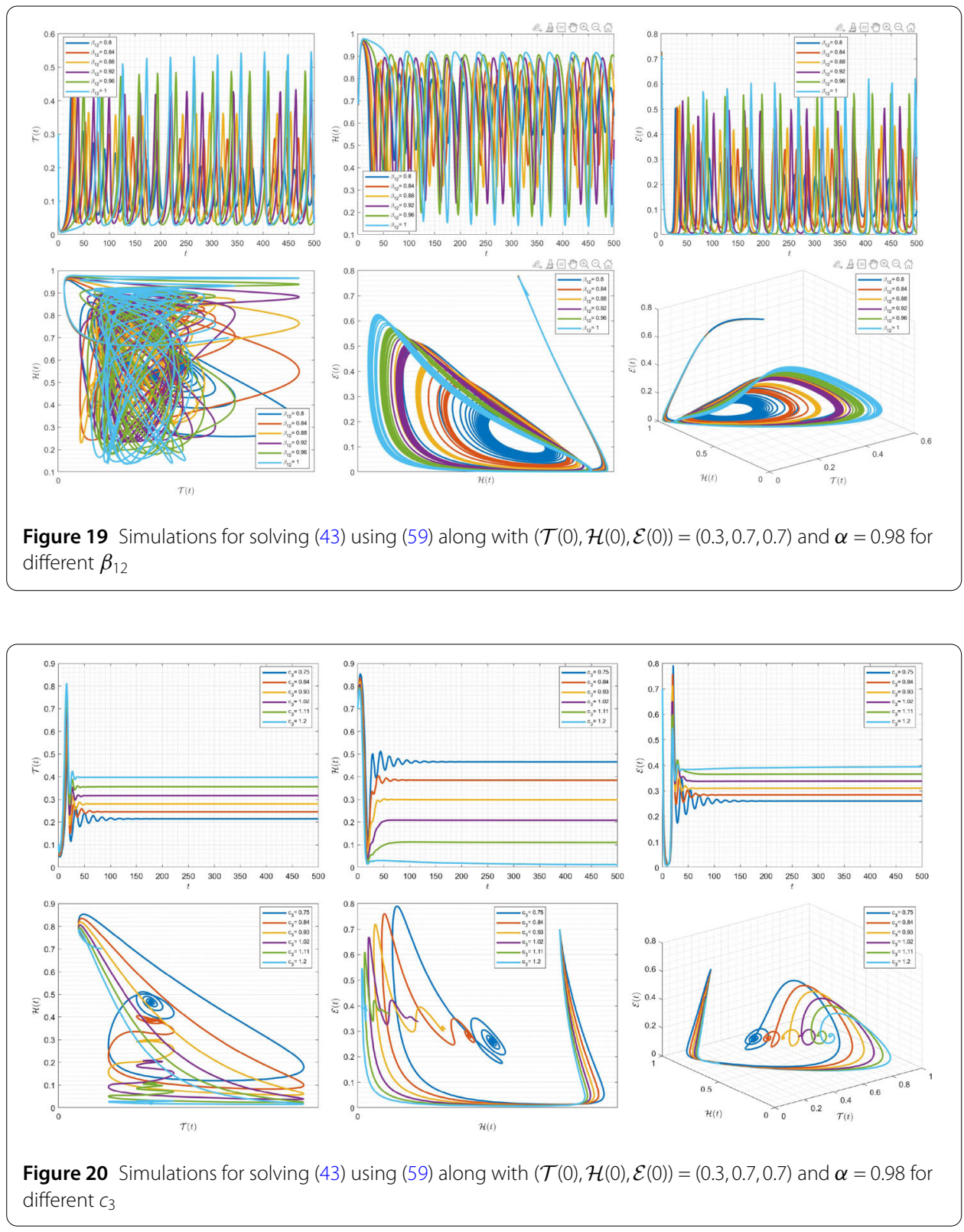

is unable to detect and attack cancer cells, then effective treatment must be used to control the tumor growth.

\section{Conclusion}

Recent advances in the presentation of efficient numerical methods in fractional differential calculus are a great help to researchers in modeling real phenomena. It has also been proven that differential calculus of integer order in some cases is incapable of describing the behavior of some phenomena or faces fundamental problems. In this paper, we study the dynamics of a tumor-immune model due to the unpredictable growth of tumor cells described by an attractive form of nonlinear differential equations. The importance of this model has led to many different approaches of studying the problem. Our study in the paper makes it clear how tumor cells interact with the immune system. The main dif- 
ference of this paper from other papers on this system is that we have used new fractional and fractal-fractional definitions for the derivative in the system structure. Some of these new concepts of differentiation were introduced in 2017 by Atangana and his collaborators. They combine the idea of fractal derivative and fractional differentiation, which takes into account the memory, fractal effect, and nonlocality. The model considers processes like power-law, fading memory, and crossover. It is important to note that the numerical methods and analysis used in this paper are quite different from those presented in [24]. In fact, this work can be considered as a complement to the content presented in this paper. The basis of these mathematical algorithms is applying some fundamental axioms of fractional derivative and the first-order interpolation. The existence and uniqueness of the model solutions were also investigated. The interesting attractors obtained in this paper imply that these new fractional and fractal-fractional operators can describe newer aspects of the behavior of these systems than fractional derivatives. Some of these features cannot be described by conventional integer-order operators. Through numerical simulations, we have confirmed the chaotic dynamics of the model by taking certain parameters in the model and suitable initial conditions. These results indicate that the values considered for the fractional-order derivatives significantly influence the dynamic behavior of the problem. The fractal-fractional operators allow us to describe self-similar problems with power-law, fading memory, and crossover behavior. In addition, the chaotic behavior seen in the results is entirely consistent with the inherent nature of the problem. These systems are recognized as complex real-world problems that cannot be represented with classical or fractional differential operators. Numerical simulations confirm that a change in fractional order exhibits memory properties and very strange complex dynamical behaviors. The results of this paper show that other similar problems in this field can be described and investigated using the numerical methods used in this paper.

\author{
Acknowledgements \\ Not applicable. \\ Funding \\ This research work is not supported by any funding agencies. \\ Availability of data and materials \\ Not applicable. \\ Competing interests \\ The author declares that he has no competing interests. \\ Authors' contributions \\ All authors read and approved the final manuscript.
}

\title{
Publisher's Note
}

Springer Nature remains neutral with regard to jurisdictional claims in published maps and institutional affiliations.

Received: 7 September 2020 Accepted: 8 October 2020 Published online: 19 October 2020

References

1. Agrawal, O.P.: Fractional variational calculus in terms of Riesz fractional derivatives. J. Phys. A, Math. Theor. 40(24), 6287 (2007)

2. Alkahtani, B.S.T:: Chua's circuit model with Atangana-Baleanu derivative with fractional order. Chaos Solitons Fractals 89, 547-551 (2016)

3. Ageel, M., Ahmad, S.: Analytical and numerical study of Hopf bifurcation scenario for a three-dimensional chaotic system. Nonlinear Dyn. 84(2), 755-765 (2016)

4. Atangana, A.: Fractal-fractional differentiation and integration: connecting fractal calculus and fractional calculus to predict complex system. Chaos Solitons Fractals 102, 396-406 (2017) 
5. Atangana, A.: Non validity of index law in fractional calculus: a fractional differential operator with Markovian and non-Markovian properties. Phys. A, Stat. Mech. Appl. 505, 688-706 (2018)

6. Atangana, A., Akgül, A.: Can transfer function and Bode diagram be obtained from Sumudu transform. Alex. Eng. J. 59(4), 1971-1984 (2020)

7. Atangana, A., Araz, S.I.: Atangana-Seda numerical scheme for labyrinth attractor with new differ. Geophys. J. Int. 13(5), 529-539 (2020)

8. Atangana, A., Araz, S.I.: New numerical approximation for Chua attractor with fractional and fractal-fractional operators. Alex. Eng. J. 59(5), 3275-3296 (2020)

9. Atangana, A., Araz, S.l.: New numerical method for ordinary differential equations: Newton polynomial. J. Comput. Appl. Math. 372, 112622 (2020)

10. Atangana, A., Baleanu, D.: New fractional derivatives with nonlocal and non-singular kernel: theory and application to heat transfer model (2016). arXiv:1602.03408. arXiv preprint

11. Atangana, A., Qureshi, S.: Modeling attractors of chaotic dynamical systems with fractal-fractional operators. Chaos Solitons Fractals 123, 320-337 (2019)

12. Baker, G.L., Baker, G.L., Gollub, J.P.: Chaotic Dynamics: An Introduction. Cambridge University Press, Cambridge (1996)

13. Caputo, M., Fabrizio, M.: A new definition of fractional derivative without singular kernel. Prog. Fract. Differ. Appl. 1(2), $1-13(2015)$

14. Caputo, M., Mainardi, F.: A new dissipation model based on memory mechanism. Pure Appl. Geophys. 91(1), 134-147 (1971)

15. Diethelm, K.: The Analysis of Fractional Differential Equations: An Application-Oriented Exposition Using Differential Operators of Caputo Type. Springer, Berlin (2010)

16. Gao, W., Veeresha, P., Prakasha, D., Baskonus, H.M., Yel, G.: New approach for the model describing the deathly disease in pregnant women using Mittag-Leffler function. Chaos Solitons Fractals 134, 109696 (2020)

17. Ghanbari, B., Atangana, A.: A new application of fractional Atangana-Baleanu derivatives: designing ABC-fractional masks in image processing. Phys. A, Stat. Mech. Appl. 542, 123516 (2020)

18. Ghanbari, B., Atangana, A.: Some new edge detecting techniques based on fractional derivatives with non-local and non-singular kernels. Adv. Differ. Equ. 2020(1), 1 (2020)

19. Ghanbari, B., Cattani, C.: On fractional predator and prey models with mutualistic predation including non-local and nonsingular kernels. Chaos Solitons Fractals 136, 109823 (2020)

20. Ghanbari, B., Kumar, D.: Numerical solution of predator-prey model with Beddington-DeAngelis functional response and fractional derivatives with Mittag-Leffler kernel. Chaos, Interdiscip. J. Nonlinear Sci. 29(6), 063103 (2019)

21. Ghanbari, B., Nisar, K.S.: Some effective numerical techniques for chaotic systems involving fractal-fractional derivatives with different laws. Front. Phys. 8, 192 (2020) https://www.frontiersin.org/article/10.3389/fphy.2020.00192. https://doi.org/10.3389/fphy.2020.00192

22. Gómez-Aguilar, J.: Chaos and multiple attractors in a fractal-fractional Shinriki's oscillator model. Phys. A, Stat. Mech. Appl. 539, 122918 (2020)

23. Gómez-Aguilar, J.: Multiple attractors and periodicity on the Vallis model for El Niño/La Niña-Southern oscillation model. J. Atmos. Sol.-Terr. Phys. 197, 105172 (2020)

24. Gómez-Aguilar, J.F., López-López, M.G., Alvarado-Martínez, V.M., Baleanu, D., Khan, H.: Chaos in a cancer model via fractional derivatives with exponential decay and Mittag-Leffler law. Entropy 19(12), 681 (2017)

25. Hoan, L.V.C., Akinlar, M.A., Inc, M., Gómez-Aguilar, J., Chu, Y.M., Almohsen, B.: A new fractional-order compartmental disease model. Alex. Eng. J. 59(5), 3187-3196 (2020)

26. Itik, M., Banks, S.P.: Chaos in a three-dimensional cancer model. Int. J. Bifurc. Chaos 20(1), 71-79 (2010)

27. Jajarmi, A., Ghanbari, B., Baleanu, D.: A new and efficient numerical method for the fractional modeling and optimal control of diabetes and tuberculosis co-existence. Chaos, Interdiscip. J. Nonlinear Sci. 29(9), 093111 (2019)

28. Khajanchi, S., Perc, M., Ghosh, D.: The influence of time delay in a chaotic cancer model. Chaos, Interdiscip. J. Nonlinear Sci. 28(10), 103101 (2018)

29. Khan, A., Abdeljawad, T., Gómez-Aguilar, J., Khan, H.: Dynamical study of fractional order mutualism parasitism food web module. Chaos Solitons Fractals 134, 109685 (2020)

30. Kilbas, A.A., Srivastava, H.M., Trujillo, J.J.: Theory and Applications of Fractional Differential Equations, vol. 204. Elsevier, Amsterdam (2006)

31. Li, C., Tao, C.: On the fractional Adams method. Comput. Math. Appl. 58(8), 1573-1588 (2009)

32. López, A.G., Sabuco, J., Seoane, J.M., Duarte, J., Januário, C., Sanjuán, M.A.: Avoiding healthy cells extinction in a cancer model. J. Theor. Biol. 349, 74-81 (2014)

33. Losada, J., Nieto, J..: Properties of a new fractional derivative without singular kernel. Prog. Fract. Differ. Appl. 1(2), 87-92 (2015)

34. Medio, A., Gallo, G., et al.: Chaotic Dynamics. Cambridge Books (1995)

35. Naik, P.A., Zu, J., Owolabi, K.M.: Global dynamics of a fractional order model for the transmission of HIV epidemic with optimal control. Chaos Solitons Fractals 138, 109826 (2020)

36. Parker, T.S., Chua, L.: Practical Numerical Algorithms for Chaotic Systems. Springer, Berlin (2012)

37. Qureshi, S., Atangana, A.: Fractal-fractional differentiation for the modeling and mathematical analysis of nonlinear diarrhea transmission dynamics under the use of real data. Chaos Solitons Fractals 136, 109812 (2020)

38. Rasband, S.N.: Chaotic Dynamics of Nonlinear Systems. Courier Dover Publications (2015)

39. Sene, N.: Sir epidemic model with Mittag-Leffler fractional derivative. Chaos Solitons Fractals 137, 109833 (2020)

40. Starkov, K.E., Coria, L.N.: Global dynamics of the Kirschner-Panetta model for the tumor immunotherapy. Nonlinear Anal., Real World Appl. 14(3), 1425-1433 (2013)

41. Valle, P.A., Coria, L.N., Gamboa, D., Plata, C.: Bounding the dynamics of a chaotic-cancer mathematical model. Math. Probl. Eng. 2018, Article ID 9787015 (2018)

42. Wei, H.C., Lin, J.T.: Periodically pulsed immunotherapy in a mathematical model of tumor-immune interaction. Int. J. Bifurc. Chaos 23(4), 1350068 (2013) 